\title{
Robustness Analysis for Decision Under Uncertainty with Rule-based Preference Model
}

\author{
Miłosz Kadziński ${ }^{\mathrm{a}}$, Roman Słowiński ${ }^{\mathrm{a}, \mathrm{c}, *}$, Salvatore Greco ${ }^{\mathrm{b}, \mathrm{d}}$ \\ ${ }^{a}$ Institute of Computing Science, Poznań University of Technology, Poznań, Poland \\ ${ }^{b}$ Department of Economics and Business, University of Catania, Catania, Italy \\ ${ }^{c}$ Systems Research Institute, Polish Academy of Sciences, Warsaw, Poland \\ ${ }^{d}$ University of Portsmouth, Portsmouth Business School, Centre of Operations Research and Logistics (CORL), \\ Richmond Building, Portland Street, Portsmouth PO1 3DE, United Kingdom
}

\begin{abstract}
We consider decision under uncertainty as a multi-attribute classification problem where a set of acts is described by outcomes gained with given probabilities. The Decision Maker (DM) provides desired classification for a small subset of reference acts. Such preference information is structured using Dominance-based Rough Set Approach (DRSA), and the resulting lower approximations of the quality class unions are used as an input for construction of an aggregated preference model. We induce all minimal-cover sets of rules being compatible with the non-ambiguous assignment examples, and satisfying some additional requirements that may be imposed by the DM. Applying such compatible instances of the preference model on a set of all acts, we draw conclusions about the certainty of recommendation assured by different minimal-cover sets of rules. In particular, we analyze the diversity of class assignments, assignment-based preference relations, and class cardinalities. Then, we solve an optimization problem to get a univocal (precise) classification for all acts, taking into account the robustness concern. This optimization problem admits incorporation of additional indirect and imprecise preferences in form of desired class cardinalities and assignment-based pairwise comparisons. Finally, we extend the proposed approach to group decision under uncertainty. We present a set of indicators and outcomes for judging the spaces of consensus and disagreement between the DMs.
\end{abstract}

Keywords: Decision under uncertainty, Classification, Dominance-based Rough Set Approach, Robustness Analysis, Univocal recommendation, Group decision

\section{Introduction}

Decision under uncertainty is a classical topic of decision theory (see [11] for a review). In this case, the Decision Maker (DM) considers a set of acts whose consequences are uncertain. There are

${ }^{*}$ Corresponding author: Institute of Computing Science, Poznań University of Technology, Piotrowo 2, 60-965 Poznań, Poland. Tel. +48-61 6652922.

Email addresses: milosz.kadzinski@cs.put.poznan.pl (Miłosz Kadziński), roman.slowinski@cs.put.poznan.pl (Roman Słowiński), salgreco@unict.it (Salvatore Greco)

This paper significantly extends [37] which won the Best Paper Award at the 2014 Joint Rough Set Symposium (JRS 2014) 
many possible states of the world with given probabilities. Depending on the actual state, an act can yield a corresponding outcome with a given probability. It is assumed, moreover, that the DM is able to express preferences with respect to the outcomes predicted for the considered acts with given probabilities. The preference information provided by the DM takes part in the construction of her/his preference model. This model induces a preference relation in the set of acts, richer than the stochastic dominance relation [44]. Its proper exploitation leads to a recommendation in terms of choice, ranking, or classification.

The main approaches to modeling decision under uncertainty are based on the expected utility theory, which was axiomatized by von Neumann and Morgenstern [43] for objective probability, and by Savage [33] for subjective probability. Many experiments showed, however, systematic violation of the expected utility hypotheses (see, e.g., [1], [10], [26]). In consequence, many alternative models weakening some original axioms have been proposed (see [38] for a review). In this context, it is worth mentioning the work on ambiguity as a source of uncertainty [29]. Furthermore, Greco, Matarazzo, and Słowiński [16] proposed an approach to decision under uncertainty based on stochastic dominance, which is the weakest assumption possible. They adapted for this their Dominance-based Rough Set Approach (DRSA) [14], which extends the rough set concept introduced by Pawlak [30] by handling ambiguity with respect to dominance (for some recent advances in DRSA, see, e.g., $[22,28,36,39,40,45])$.

In the approach presented in [16], the decision under uncertainty is formulated in terms of a multiattribute classification problem. The set of classified objects is the set of acts and the set of condition attributes describing the acts is the set of probabilities derived from an additive probability distribution defined over disjoint and exhaustive states of the world. The method expects the DM to assign a small subset of acts, called reference acts, to some pre-defined classes of overall quality. Precisely, each assignment of a reference act to a quality class is a classification example characterized by the outcomes for a finite set of given probabilities (condition attributes), and by the assignment to one of several classes of overall quality (decision attribute). The set of classification examples constitutes preference information conditioned by the value system of the DM. Such classification data is structured using DRSA, and then a set of decision rules is induced. These rules explain the preferences of the DM in terms of conditions on values of outcomes for particular probabilities, that ensure assignment of reference acts to so-called upward union or downward union of classes. A set of rules covering the classification examples constitutes a preference model of the DM. It is subsequently used to classify the non-reference acts. An intuitive principle which guides this approach can be formulated as follows: "the more and the more probable, the better".

When applying DRSA to decision under uncertainty in the way suggested in [16], one needs to be aware of an important limitation of this approach. When classifying the non-reference acts, the authors of [16] use just a single set of rules. However, such representation of the DM preferences is not unique, because, in general, there may exist many sets of rules that are compatible with the provided preference information. Thus, choosing among them is to a large extent arbitrary. Moreover, when applied on the non-reference acts, different sets of rules may suggest different class assignments for 
the same acts. As explained in [6], existence of alternative instances of a preference model - all of them being compatible with the input preference information - requires robustness analysis of the recommendations delivered by these instances.

Addressing the above mentioned drawback, we proposed a two-fold revision of DRSA to decision under uncertainty [37]. On the one hand, we considered all minimal-cover sets of rules as compatible instances of the preference model. Adopting this strategy, we avoided arbitrary selection of one among many sets of rules which reproduce equally well the provided preference information (classification examples). On the other hand, we investigated the diversity of the recommendations suggested by these sets by producing two types of assignment for each act. The possible assignment holds if and only if it is confirmed by at least one compatible minimal-cover set of rules, whereas the necessary assignment needs to be supported by all minimal-cover sets of rules. In this way, we adapted a more general principle of Robust Ordinal Regression (ROR) [6] to decision under uncertainty formulated as a multi-attribute classification problem. The aim of this paper is to extend this approach by proposing some advanced methods for robustness analysis in the context of decision under uncertainty (for other applications of ROR to ordinal classification (sorting) problems, see [18, 24, 25]).

First, we adapt an integrated framework for robustness analysis in multiple criteria sorting problem [20] to DRSA. Thus, when investigating the stability of the delivered recommendation, apart from considering class assignments for each act individually, we will take into account two other perspectives. On the one hand, we will refer to the assignment-based preference relation which holds for an ordered pair of acts if one of them is assigned to a class at least as good as the other [13, 24]. Such a relation is called an assignment-based outranking relation. On the other hand, we will compute cardinalities of the quality classes. The basic analysis consists in considering the necessary, possible, or extreme outcomes. However, since all compatible minimal-cover sets of rules are known, we are able to compute the assignment-based outranking indices or class cardinality indices defined as the shares of compatible minimal-cover sets of rules which confirm some classification result. Overall, the DM may observe the impact of her/his preference information on the recommendation concerning not only the whole set of acts, but also all pairs of acts and all quality classes.

Second, we exploit the results of robustness analysis to construct a univocal recommendation that would suggest assignment of each act to a single class. For this purpose, we analyze the cumulative class acceptability indices reflecting the shares of minimal-cover sets of rules that assign an act to each class. A natural proposal consists in selecting for each act a class with the maximal acceptability. However, we extend this basic proposal by accounting for additional types of indirect and imprecise preference information to be taken into account when constructing a univocal recommendation. These are desired class cardinalities and assignment-based pairwise comparisons. The former specify the minimal and/or maximal number of acts that can be assigned to each act [23], while the latter indicate imprecise comparison between desired classes for pair of acts, but without specifying any concrete classes [20]. Note that the proposed procedure is more general, being independent of the method used for robustness analysis. Thus, it is equally desirable for use with some value- [24] or outranking-based [41] stochastic approaches for Multiple Criteria Decision Aiding (MCDA). 
Third, we extend DRSA for decision under uncertainty to group decision $[4,15]$. In this approach, each DM provides her/his individual preference information. Then, the collective results account for the robustness analysis conducted for each DM individually. In this way, we avoid discussions of DMs on technical parameters, providing instead a set of indicators and outcomes that inform to what extent a group of DMs agrees or disagrees about the assignment of all acts. These results can be used by an analyst for organizing a discussion focused on reaching an agreement between the DMs.

Fourth, the approach proposed in [37] is based on considering all minimal-cover sets of rules compatible with the DM's preferences. We show how it can be extended to construct all satisfactory sets of decision rule which are consistent with some user's requirements. These may concern, e.g., the minimal support of rules, non-redundant covering of a set of reference acts, or the maximal number of condition attributes to be used in the set of rules.

Finally, although the work in [37] referred only to an additive probability distribution defined over events (i.e., subsets of states of the world), we show that it can be used together with a non-additive probability distribution, or even a probability with an ordinal qualitative scale. This advantage derives from the intrinsic feature of DRSA which takes into account the ordinal properties of a probability only.

The paper is organized as follows. Section 2 recalls basics of DRSA for decision under uncertainty. Section 3 describes how decision aiding can be performed with the proposed approach. Then, we recall the algorithms for generating all compatible rules and all compatible minimal-cover sets of rules in Sections 4 and 5, respectively. We also discuss how to generate satisfactory rules and satisfactory sets of rules. Section 6 is devoted to an integrated framework for robustness analysis. In Section 7, we discuss procedures for constructing a univocal classification while taking into account the robustness concern and additional indirect preference information. Section 8 is devoted to group decision under uncertainty. Section 9 concludes the paper.

\section{Dominance-based Rough Set Approach for Decision Under Uncertainty}

Following [16] and [37], we use the following notation:

- a finite set $S=\left\{s_{1}, s_{2}, \ldots, s_{u}\right\}$ of states of the world, or simply states, which are disjoint and exhaustive;

- an event $W$ corresponding to a subset of states, $W \subseteq S$;

- an a priori probability distribution $P$ over $S$ : more precisely; the probabilities of states $s_{1}, s_{2}$, $\ldots, s_{u}$ are given by $p_{1}, p_{2}, \ldots, p_{u}$, respectively, $p_{1}+p_{2}+\ldots+p_{u}=1, p_{i} \geq 0, i=1, \ldots, u$;

- $\Pi$ is a set of all different probabilities of the events;

- a set $A=\left\{a_{1}, a_{2}, \ldots, a_{m}\right\}$ of all considered acts, and a set $A^{R} \subset A$ of reference acts, for which the DM expresses desired assignments (decision examples); 
- a set $X=\left\{x_{1}, x_{2}, \ldots, x_{r}\right\}$ of possible outcomes; for the sake of simplicity, we assume they are expressed in the monetary terms $(X \subseteq \mathbb{R})$;

- a function $g: A \times S \rightarrow X$ assigning to each pair act-state $\left(a_{i}, s_{j}\right) \in A \times S$ an outcome $x_{k} \in X$;

- a set of quality classes $\boldsymbol{C l}=\left\{C l_{1}, C l_{2}, \ldots, C l_{n}\right\}$, such that $C l_{1} \cup C l_{2} \cup \ldots \cup C l_{n}=A^{R}$, $C l_{r} \cap C l_{q}=\emptyset$ for each $r, q \in H=\{1, \ldots, n\}$ with $r \neq q$; the classes from $\boldsymbol{C l}$ are preferenceordered according to the increasing order of their indices;

- a function $e: A^{R} \rightarrow \boldsymbol{C l}$ assigning each act $a_{i} \in A^{R}$ to a quality class $C l_{j} \in \boldsymbol{C l}$.

The rest of notation used throughout the paper is summarized in Appendix A. In what follows, we recall the basic concepts of DRSA for decision under uncertainty.

Stochastic dominance. The sole information coming out from the analysis of the outcomes attained by the acts in multiple states of the world is the stochastic dominance relation. When considering acts $a_{p}, a_{q} \in A, a_{p}$ stochastically dominates $a_{q}$ if for each outcome $x \in X, a_{p}$ gives an outcome at least as good as $x$ with a probability at least as great as the probability that $a_{q}$ gives the same outcome $[27,32,44]$, i.e., for all $x \in X$,

$$
P\left[S\left(a_{p}, x\right)\right] \geq P\left[S\left(a_{q}, x\right)\right]
$$

where, for each $\left(a_{i}, x\right) \in A \times X, S\left(a_{i}, x\right)=\left\{s_{j} \in S: g\left(a_{i}, s_{j}\right) \geq x\right\}$.

Probability distribution over events. On the basis of $P$, we can assign to each subset of states $W \subseteq S$, which corresponds to an event, the probability $P(W)$ that one of the states in $W$ is verified, i.e., $P(W)=\sum_{i: s_{i} \in W} p_{i}$, and then we can build up the set $\Pi$ of all possible values $P(W)$, i.e.:

$$
\Pi=\{\pi \in[0,1]: \pi=P(W), W \subseteq S\}
$$

Probability of yielding a given outcome by an act. Let us define the following functions $z: A \times S \rightarrow \Pi$ and $z^{\prime}: A \times S \rightarrow \Pi$ assigning to each act-state pair $\left(a_{i}, s_{j}\right) \in A \times S$ a probability $\pi \in \Pi$, as follows:

$$
z\left(a_{i}, s_{j}\right)=\sum_{r: g\left(a_{i}, s_{r}\right) \geq g\left(a_{i}, s_{j}\right)} p_{r} \quad \text { and } \quad z^{\prime}\left(a_{i}, s_{j}\right)=\sum_{r: g\left(a_{i}, s_{r}\right) \leq g\left(a_{i}, s_{j}\right)} p_{r} .
$$

Therefore, $z\left(a_{i}, s_{j}\right)\left(z^{\prime}\left(a_{i}, s_{j}\right)\right)$ represents the probability of obtaining an outcome whose value is at least (at most) $g\left(a_{i}, s_{j}\right)$ by act $a_{i}$.

On the basis of functions $z\left(a_{i}, s_{j}\right)$ and $z^{\prime}\left(a_{i}, s_{j}\right)$, we can define functions, respectively, $\rho: A \times \Pi$ $\rightarrow X$ and $\rho^{\prime}: A \times \Pi \rightarrow X$ as follows:

$$
\rho\left(a_{i}, \pi\right)=\max _{j: z\left(a_{i}, s_{j}\right) \geq \pi}\left\{g\left(a_{i}, s_{j}\right)\right\} \quad \text { and } \quad \rho^{\prime}\left(a_{i}, \pi\right)=\min _{j: z^{\prime}\left(a_{i}, s_{j}\right) \geq \pi}\left\{g\left(a_{i}, s_{j}\right)\right\} \text {. }
$$


Thus, $\rho\left(a_{i}, \pi\right)=x\left(\rho^{\prime}\left(a_{i}, \pi\right)=x\right)$ means that the outcome got by act $a_{i}$ is greater (smaller) than or equal to $x$ with a probability at least $\pi$. As observed in [16], information given by $\rho\left(a_{i}, \pi\right)$ and $\rho^{\prime}\left(a_{i}, \pi\right)$ is related such that for all $a_{i} \in A$ and $\pi_{(j-1)}, \pi_{(j)} \in \Pi$ :

$$
\rho\left(a_{i}, \pi_{(j)}\right)=\rho^{\prime}\left(a_{i}, 1-\pi_{(j-1)}\right)
$$

where the probabilities $\pi_{(j-1)}, \pi_{(j)}$ are two consecutive probabilities from an increasing order of all probabilities from $\Pi$, i.e., $\pi_{(j-1)} \leq \pi_{(j)}$. This implies that the analysis of the possible decisions can be equivalently conducted using either $\rho\left(a_{i}, \pi\right)$ or $\rho^{\prime}\left(a_{i}, \pi\right)$.

Therefore, in the context of stochastic acts, if we need to express an outcome in positive terms, we refer to $\rho(a, \pi)$ giving a lower bound of an outcome ("for act $a$ there is a probability $\pi$ to gain at least $\rho(a, \pi)$ "), while if we need to express an outcome in negative terms, we refer to $\rho^{\prime}(a, \pi)$ giving an upper bound of an outcome ("for act $a$ there is a probability $\pi$ to gain at most $\rho^{\prime}(a, \pi)$ ").

Given $a_{p}, a_{q} \in A, a_{p}$ stochastically dominates $a_{q}$ if and only if $\rho\left(a_{p}, \pi\right) \geq \rho\left(a_{q}, \pi\right)$ for each $\pi \in \Pi$. This is equivalent to say: given $a_{p}, a_{q} \in A, a_{p}$ stochastically dominates $a_{q}$ if and only if $\rho^{\prime}\left(a_{p}, \pi\right) \geq \rho^{\prime}\left(a_{q}, \pi\right)$ for each $\pi \in \Pi$.

Decision under uncertainty as a multi-attribute classification problem. The above considerations lead us to formulation of the decision under uncertainty in terms of a multi-attribute classification problem, where the set of objects to be classified is the set of acts $A$ described by the set of condition attributes specifying the outcomes $\rho\left(a_{i}, \pi\right)$ (or $\left.\rho^{\prime}\left(a_{i}, \pi\right)\right)$ for all $\pi \in \Pi$. The set $X$ is a value set of the condition attributes.

Classification examples. The classification (assignment) examples concerning the reference acts $A^{R} \subset A$ constitute the DM's preference information considered in the context of decision under uncertainty. Formally, they are presented as an information table whose rows correspond to the reference acts belonging to set $A^{R}$, and columns correspond to the condition attributes from set $\Pi$, and to the decision attribute $\boldsymbol{c l}$ assigning the acts from $A^{R}$ to the classes from $\boldsymbol{C l}$. The entries of the information table are values of an information function $\boldsymbol{f}\left(a_{i}, \pi\right)=\rho\left(a_{i}, \pi\right)$ and $\boldsymbol{f}\left(a_{i}, \boldsymbol{c l}\right)=e\left(a_{i}\right)$. Let us observe that due to the above stated equivalence, one can consider alternatively an information function $\boldsymbol{f}^{\prime}\left(a_{i}, \pi\right)=\rho^{\prime}\left(a_{i}, \pi\right)$.

Rough approximations of the class unions. The stochastic dominance principle says that act $a_{i}$, whose outcomes are not worse than outcomes of act $a_{j}$ for the corresponding probabilities $\left(a_{i}\right.$ stochastically dominates $\left.a_{j}\right)$, should not be assigned to a worse quality class than $a_{j}$. To handle ambiguity with respect to the stochastic dominance, we structure the classification data using DRSA. In DRSA, we are approximating the upward $C l_{\bar{t}}^{\geq}=\bigcup_{s \geq t} C l_{s}$ and downward $C l_{t}^{\leq}=\bigcup_{s \leq t} C l_{s}$, unions of classes, $t=1, \ldots, n$, using dominance cones defined in the condition attribute space for any subset of condition attributes $\Theta \subseteq \Pi$. The fact that act $a_{p}$ stochastically dominates act $a_{q}$ with respect to 
$\Theta \subseteq \Pi$ (i.e., $\rho\left(a_{p}, \pi\right) \geq \rho\left(a_{q}, \pi\right)$ for each $\left.\pi \in \Theta\right)$ is denoted by $x D_{\Theta} y$. Given $\Theta \subseteq \Pi$ and $a_{i} \in A^{R}$, the cones of dominance are:

- a set of acts dominating $a_{i}: D_{\Theta}^{+}\left(a_{i}\right)=\left\{a_{j} \in A^{R}: a_{j} D_{\Theta} a_{i}\right\}$;

- a set of acts dominated by $a_{i}: D_{\Theta}^{-}\left(a_{i}\right)=\left\{a_{j} \in A^{R}: a_{i} D_{\Theta} a_{j}\right\}$.

With respect to $\Theta \subseteq \Pi$, the set of all acts belonging to $C l_{t}^{\geq}\left(C l_{t}^{\leq}\right)$without any ambiguity constitutes the $\Theta$-lower approximation of $C l_{t}^{\geq}\left(C l_{t}^{\leq}\right)$, denoted by $\underline{\Theta}\left(C l_{t}^{\geq}\right)\left(\underline{\Theta}\left(C l_{t}^{\leq}\right)\right)$, and the set of all acts that could belong to $C l_{\bar{t}}^{\geq}\left(C l_{t}^{\leq}\right)$constitutes the $\Theta$-upper approximation of $C l_{t}^{\geq}\left(C l_{t}^{\leq}\right)$, denoted by $\bar{\Theta}\left(C l_{t}^{\geq}\right)$ $\left(\bar{\Theta}\left(C l_{t}^{\leq}\right)\right)$, i.e., for $t=1, \ldots, n$ :

$$
\begin{aligned}
& \underline{\Theta}\left(C l_{t}^{\geq}\right)=\left\{a \in A^{R}: D_{\Theta}^{+}(a) \subseteq C l_{t}^{\geq}\right\} \text {and } \underline{\Theta}\left(C l_{t}^{\leq}\right)=\left\{a \in A^{R}: D_{\Theta}^{-}(a) \subseteq C l_{t}^{\leq}\right\}, \\
& \bar{\Theta}\left(C l_{t}^{\geq}\right)=\left\{a \in A^{R}: D_{\Theta}^{-}(a) \cap C l_{t}^{\geq} \neq \emptyset\right\} \text { and } \bar{\Theta}\left(C l_{t}^{\leq}\right)=\left\{a \in A^{R}: D_{\Theta}^{+}(a) \cap C l_{t}^{\leq} \neq \emptyset\right\} .
\end{aligned}
$$

For definitions of the $\Theta$-boundaries, quality of approximation, reducts, and core, see [16, 37].

\section{Illustrative study: formulation of the decision problem and structuring the classification examples with DRSA. The following example illustrates the approach. Let us consider:}

- a set $S=\left\{s_{1}, s_{2}, s_{3}\right\}$ of states of the world;

- an a priori probability distribution $P$ over $S$ defined as follows: $p_{1}=0.20, p_{2}=0.35, p_{3}=0.45$;

- a set $A=\left\{a_{1}, \ldots, a_{20}\right\}$ of acts, and a set of reference acts $A^{R}=\left\{a_{1}, a_{2}, a_{3}, a_{7}, a_{13}, a_{18}\right\}$;

- a set $X=\{0,5,10,15,20,25,30\}$ of possible outcomes;

- a set of quality classes $\boldsymbol{C l}=\left\{C l_{1}, C l_{2}, C l_{3}\right\}$, where $C l_{1}$ is the set of bad acts, $C l_{2}$ is the set of medium acts, and $\mathrm{Cl}_{3}$ is the set of good acts;

- a function $g: A \times S \rightarrow X$ assigning to each act-state pair $\left(a_{i}, s_{j}\right) \in A \times S$ an outcome $x \in X$, and a function $e: A^{R} \rightarrow \boldsymbol{C l}$ assigning each reference act $a_{i} \in A^{R}$ to class $C l_{j} \in \boldsymbol{C l}$, presented in Table 1a).

Table $1 \mathrm{~b})$ shows the values of function $\rho\left(a_{i}, \pi\right)$, when using an additive probability distribution $P$. Let us provide some examples of the interpretation of the values in this table. Considering the row of act $a_{1}$, we have that by act $a_{1}$ the value 30 (25) in the column corresponding to $0.20(0.35)$ means that the outcome is at least 30 (25) with probability $\pi$ of at least $0.20(0.35)$. In the column corresponding to probability $\pi=0.65$, the value of 20 (15) relative to $a_{3}\left(a_{4}\right)$ means that by act $a_{3}$ $\left(a_{4}\right)$ the outcome is at least $20(15)$ with probability $\pi$ of at least 0.65 . 
Table 1: a) Acts, consequences, and assignment to classes from $\boldsymbol{C l}$ by the DM. b) Acts, values of function $\rho\left(a_{i}, \pi\right)$ and assignment to classes from $\boldsymbol{C l}$.

\begin{tabular}{|c|c|c|c|c|c|c|c|c|c|c|c|c|}
\hline & & Part a) & & & & & & t b) & & & & \\
\hline$s_{1}$ & $s_{2}$ & $s_{3}$ & & & & & & & & & & \\
\hline 0.20 & 0.35 & 0.45 & $c l$ & $\rho\left(a_{i}, \pi\right)$ & 0.20 & 0.35 & 0.45 & 0.55 & 0.65 & 0.80 & 1.00 & $c l$ \\
\hline 30 & 10 & 25 & good (3) & $a_{1}$ & 30 & 25 & 25 & 25 & 25 & 10 & 10 & 3 \\
\hline$a_{2}$ & 10 & 20 & bad (1) & $a_{2}$ & 20 & 20 & 20 & 10 & 0 & 0 & 0 & 1 \\
\hline$a_{3}$ & 30 & 20 & good (3) & $a_{3}$ & 30 & 30 & 20 & 20 & 20 & 20 & 5 & 3 \\
\hline 15 & 5 & 20 & - & $a_{4}$ & 20 & 20 & 20 & 15 & 15 & 5 & 5 & - \\
\hline$a_{5}$ & 15 & 20 & - & $a_{5}$ & 20 & 20 & 20 & 15 & 15 & 15 & 5 & - \\
\hline 30 & 15 & 10 & - & $a_{6}$ & 30 & 15 & 15 & 15 & 10 & 10 & 10 & - \\
\hline$a_{7}$ & 20 & 10 & bad (1) & $a_{7}$ & 20 & 20 & 10 & 10 & 10 & 10 & 5 & 1 \\
\hline 20 & 0 & 15 & - & $a_{8}$ & 20 & 15 & 15 & 15 & 15 & 0 & 0 & - \\
\hline 20 & 5 & 0 & - & $a_{9}$ & 20 & 5 & 5 & 5 & 0 & 0 & 0 & - \\
\hline$a_{10}$ & 20 & 30 & - & $a_{10}$ & 30 & 30 & 30 & 20 & 20 & 20 & 10 & - \\
\hline$a_{11}$ & 0 & 20 & - & $a_{11}$ & 20 & 20 & 20 & 15 & 15 & 0 & 0 & - \\
\hline$a_{12}$ & 10 & 10 & - & $a_{12}$ & 30 & 10 & 10 & 10 & 10 & 10 & 10 & - \\
\hline 10 & 10 & 20 & medium (2) & $a_{13}$ & 20 & 20 & 20 & 10 & 10 & 10 & 10 & 2 \\
\hline$a_{14}$ & 15 & 25 & - & $a_{14}$ & 20 & 20 & 20 & 15 & 0 & 0 & 0 & - \\
\hline$a_{15}$ & 10 & 30 & - & $a_{15}$ & 30 & 30 & 30 & 30 & 30 & 10 & 10 & - \\
\hline$a_{16}$ & 15 & 25 & - & $a_{16}$ & 25 & 25 & 25 & 15 & 15 & 15 & 0 & - \\
\hline 20 & 10 & 30 & - & $a_{17}$ & 30 & 30 & 30 & 20 & 20 & 10 & 10 & - \\
\hline$a_{18}$ & 10 & 30 & medium (2) & $a_{18}$ & 30 & 30 & 30 & 10 & 10 & 10 & 5 & 2 \\
\hline$a_{19}$ & 10 & 30 & - & $a_{19}$ & 30 & 30 & 30 & 10 & 10 & 10 & 0 & - \\
\hline$a_{20}$ & 30 & 25 & - & $a_{20}$ & 30 & 30 & 25 & 25 & 25 & 25 & 15 & - \\
\hline
\end{tabular}

This information table is consistent. As a result of applying DRSA, the lower and upper approximations of upward and downward unions of classes are equal to:

$$
\begin{aligned}
& \underline{\Pi}\left(C l_{3}^{\geq}\right)=\bar{\Pi}\left(C l_{3}^{\geq}\right)=\left\{a_{1}, a_{3}\right\}, \underline{\Pi}\left(C l_{2}^{\geq}\right)=\bar{\Pi}\left(C l_{2}^{\geq}\right)=\left\{a_{13}, a_{18}, a_{1}, a_{3}\right\}, \\
& \underline{\Pi}\left(C l_{1}^{\leq}\right)=\bar{\Pi}\left(C l_{1}^{\leq}\right)=\left\{a_{2}, a_{7}\right\}, \underline{\Pi}\left(C l_{2}^{\leq}\right)=\bar{\Pi}\left(C l_{2}^{\leq}\right)=\left\{a_{13}, a_{18}, a_{2}, a_{7}\right\} .
\end{aligned}
$$

Extension to a non-additive probability distribution. Since DRSA takes into account only the ordinal properties of the probability over $S$, an additive probability $P$ can be replaced with a probability $P^{\prime}$ which is a monotonically increasing transformation of $P$ [16]. Thus, $P^{\prime}$ may be defined as the non-additive probability if only for all $R, T \subseteq S, P(R) \geq P(T)$ if and only if $P^{\prime}(R) \geq$ $P^{\prime}(T)$. In our illustrative study, such probability defined for the events needs to respect the following requirement:

$$
0.45=P^{\prime}\left(\left\{s_{3}\right\}\right)<P^{\prime}\left(\left\{s_{1}, s_{2}\right\}\right)<P^{\prime}\left(\left\{s_{1}, s_{3}\right\}\right)<P^{\prime}\left(\left\{s_{2}, s_{3}\right\}\right)<P^{\prime}\left(\left\{s_{1}, s_{2}, s_{3}\right\}\right)=1 .
$$

Note that the probability measure $P^{\prime}$ admits that $P^{\prime}(R)+P^{\prime}(T) \neq P^{\prime}(R \cup T)$ in case $R \cap T \neq \emptyset$.

Furthermore, $P^{\prime}$ may be seen as a probability defined on an ordinal qualitative scale (e.g., \{impossible, little probable, ..., strongly probable, certain\}) if for all $R, T \subseteq S, P(R) \geq P(T)$ if and only if $P^{\prime}(R) \unrhd P^{\prime}(T)$, where $\unrhd$ means "at least as probable as". When replacing $P$ with $P^{\prime}$, the rough approximations of quality class unions remain the same. As a result, the rules generated 
from these approximations need to be just suitably recoded in terms of using $P^{\prime}$ rather than $P$.

Let us also note that due to the intrinsic characteristic of DRSA our approach takes into account only ordinal properties of the outcomes. Thus, it can handle ordinal qualitative scales of the outcomes, e.g., \{none, very low, low, medium low, medium high, high, very high $\}$ instead of $\{0,5,10,15,20$, $25,30\}$.

\section{Decision Aiding with the Proposed Approach}

The decision under uncertainty considered in terms of a multi-attribute classification problem can be aided with the proposed approach through the process illustrated in Figure 3. It begins by defining the problem in Step 1. This requires specification of a set of acts $A$, a set of states of the world $S$, a priori probability distributation $P$ over $S$, and a set of quality classes $\boldsymbol{C l}$. The set of acts $A$ is described by the set of condition attributes specifying the outcomes for all states of the world $S$ and for all possible probabilities of the events (i.e., the subsets of states).

Then, in Step 2 the DM provides assignment examples for a small subset of reference acts (see Section 2). In the following step, these examples are structured using the lower and upper approximations of downward and upward class unions (see Section 2). In case of inconsistency (i.e., when $\Theta$-boundary for at least one class union is non-empty), the DM may revise her/his preference information or decide to continue the analysis.

In Step 4, we construct a set of all minimal-cover sets of rules. First, we generate all minimal decision rules from the lower approximations of class unions (see Section 4). Then, exploiting these rules, we find all minimal covers for the reference acts with Integer Linear Programming (ILP) (see Section 5). At both stages it is possible to account for the DM's requirements so that to generate only satisfactory rules or sets of rules.

In Step 5, we build a recommendation in terms of a classification (sorting) problem. First, we analyze its robustness referring to all minimal-cover sets of rules (see Section 6). We account for the three perspectives: class assignments, assignment-based preference relations, and class cardinalities. On the one hand, for each of these perspectives, we derive the robust results with Linear Programming (LP) techniques, and quantify them in terms of the possible, the necessary, and the extreme. On the other hand, we provide the acceptability indices reflecting the shares of minimal-cover sets of rules that confirm some classification result.

Apart from investigating the certainty of delivered recommendation, we construct a univocal assignment for each act by exploiting the outcomes of robustness analysis (see Section 7). Precisely, we assume that an act should be assigned to the class which is indicated by the greatest share of minimal-cover sets of rules. At this stage it is possible to account for two additional forms of DM's preference information: desired class cardinalities and assignment-based pairwise comparisons. The recommendation being consistent with these requirements is constructed using Mixed-Integer Linear Programming (MILP).

All provided results are verified in Step 6. If the DM is satisfied with the recommendation, the 


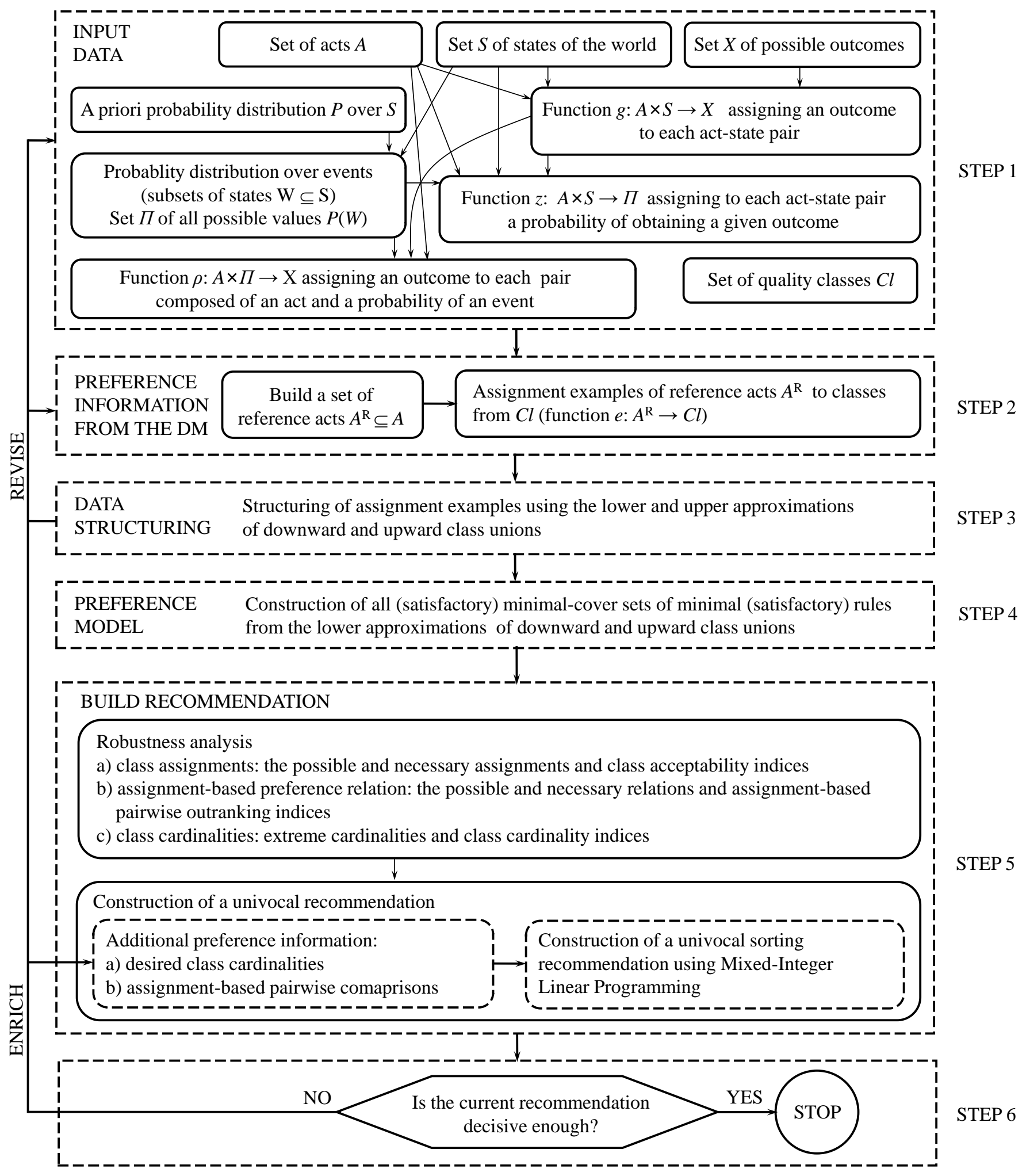

Figure 1: Decision aiding process for the proposed approach 
process ends. Otherwise, (s)he should revise or enrich the assignment examples provided in Step 2 or other forms of preference information given in Step 5.

The approach can be also used withing a group decision setting. In Section 8, we discuss some indicators that may be computed to inform a group of DMs about the spaces of disagreement with respect to the classification of all acts. These outcomes may be used to support a consensus reaching process.

\section{Generating All Compatible Minimal Rules}

The dominance-based rough approximations of upward and downward unions of classes serve to induce a generalized description of the reference acts in terms of "if . ., then ..." decision rules. The main advantage of using rules over utility function in the context of decision under uncertainty is that rules form a transparent link between the input preference information and the recommendation at the output, thus, discovering the conditions that drive DM's decision. Indeed, each rule can be interpreted as an easily understandable scenario putting together some elementary conditions (outcomes obtained with given probabilities) and a consequent (class assignment). Moreover, rules represent more complex interactions than usual additive utility function, and make non-compensatory aggregation of condition attributes [35].

In what follows, we focus on certain decision rules induced from the set of consistent reference acts. An act $a \in A^{R}$ is consistent with respect to the upward union $C l_{t}^{\geq}, t=2, \ldots, n$, if $a$ belongs to the lower approximation of $C l_{t}^{\geq}$. Analogously, an act $a \in A^{R}$ is consistent with respect to the downward union $C l_{t}^{\leq}, t=1, \ldots, n-1$, if $a$ belongs to the lower approximation of $C l_{t}^{\leq}$. For a given upward or downward union of classes, $C l_{t}^{\geq}$or $C l_{r}^{\leq}$, the decision rules induced under a hypothesis that the reference acts belonging to $\underline{\Pi}\left(C l_{t}^{\geq}\right)$or $\underline{\Pi}\left(C l_{r}^{\leq}\right)$are positive and all the other reference acts negative, suggest a certain assignment to "at least class $C l_{t}$ " or to "at most class $C l_{r}$ ", respectively. The syntax of decision rules obtained from DRSA is the following:

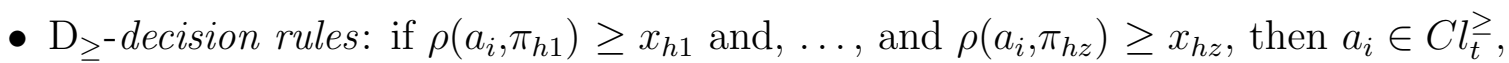
where $\pi_{h 1}, \ldots, \pi_{h z} \in \Pi, x_{h 1}, \ldots, x_{h z} \in X$, and $t \in\{2, \ldots, n\}$;

for example, when considering rule $r_{\geq 2}^{5}$ provided in Table 2 :

$$
r_{\geq 2}^{5} \equiv \text { if } \rho\left(a_{i}, 0.45\right) \geq 20 \text { and } \rho\left(a_{i}, 0.65\right) \geq 10, \text { then } a_{i} \in C l_{2}^{\geq}
$$

its interpretation is as follows: "if by act $a_{i}$ the outcome is at least 20 with probability at least 0.45 , and the outcome is at least 10 with probability at least 0.65 , then $a_{i}$ is assigned to at least class $\mathrm{Cl}_{2}$ ";

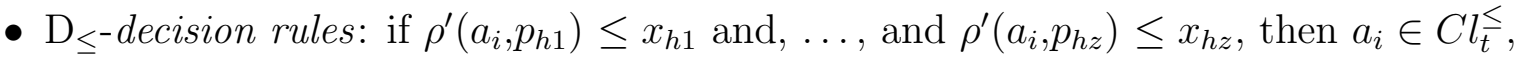
where $\pi_{h 1}, \ldots, \pi_{h z} \in \Pi, x_{h 1}, \ldots, x_{h z} \in X$, and $t \in\{1, \ldots, n-1\}$; 
for example, when considering rule $r_{\leq 1}^{3}$ provided in Table 2:

$$
r_{\leq 1}^{3} \equiv \text { if } \rho^{\prime}\left(a_{i}, 0.80\right) \leq 20 \text { and } \rho^{\prime}\left(a_{i}, 0.20\right) \leq 5 \text {, then } a \in C l_{1}^{\leq},
$$

its interpretation is as follows: "if by act $a_{i}$ the outcome is at most 20 with probability at least 0.80 , and the outcome is at most 5 with probability at least 0.20 , then $a_{i}$ is assigned to class at most $C l_{1} "$.

Let us observe that, due to the equivalence stated in Section 2, all above decision rules can be expressed equivalently in terms of values of $\rho\left(a_{i}, \pi\right)$ or $\rho^{\prime}\left(a_{i}, \pi\right)$ [16]. Nevertheless, the above proposed syntax with $\mathrm{D}_{\geq-}$decision rules expressed in terms of $\rho\left(a_{i}, \pi\right)$ and $\mathrm{D}_{\leq-}$decision rules expressed in terms of $\rho^{\prime}\left(a_{i}, \pi\right)$, is much more natural and meaningful.

In the following, we characterize an algorithm which generates all certain decision rules for $\underline{\Pi}\left(C l_{t}^{\geq}\right)$, $t=2, \ldots, n$. The algorithm for $\underline{\Pi}\left(C l_{t}^{\leq}\right), t=1, \ldots, n-1$, can be formulated analogously. We focus on induction of "mix of conditions" rules, which are possibly founded by multiple reference acts. The detailed formulation of the referred algorithms can be found in [22].

In the first phase, we generate a set of elementary conditions $C_{1}$ to be used in the construction of decision rules. This set is composed of conditions in form $\rho\left(a, \pi_{h}\right) \geq x_{h}$, such that there exists $a_{i} \in \underline{\Pi}\left(C l_{t}^{\geq}\right): \rho\left(a_{i}, \pi_{h}\right)=x_{h}$. In the second phase, we generate a set of conjunctions of elementary conditions which cover at least one reference act from $\underline{\Pi}\left(C l_{t}^{\geq}\right)$. It is an iterative process in which conjunctions of size $k+1$ are constructed from conjunctions of size $k$. Precisely, each conjunction of size $k+1$ is obtained by merging a pair of conjunctions of size $k$ which contain the same $k-1$ conditions, thus, differing by just a single elementary condition. These differentiating conditions need to concern different attributes. This procedure is repeated as long as it is possible to obtain conjunctions of a particular size. At each stage, we neglect conjunctions of size $k$ with negative support equal to 0 , since they already contain all conditions necessary to discriminate positive and negative examples. Moreover, the set $C_{k+1}$ of conjunctions of size $k+1$ contains only these conjunctions whose positive support is greater than 0 .

After generating all possible conjunctions of elementary conditions covering at least one reference act from $\underline{\Pi}\left(C l_{t}^{\geq}\right)$, we eliminate conjunctions covering any negative example from $A^{R} \backslash \underline{\Pi}\left(C l_{t}^{\geq}\right)$. Subsequently, we remove the conjunctions of conditions which are not minimal, i.e., such that there exists some other conjunction:

- using a subset of elementary conditions or/and weaker elementary conditions; for example, when considering three rules, $\mathrm{r} 1 \equiv$ if $\rho\left(a_{i}, 0.65\right) \geq 15$, then $a_{i} \in C l_{2}^{\geq}$, and $\mathrm{r} 2 \equiv$ if $\rho\left(a_{i}, 0.65\right) \geq$ 25 , then $a_{i} \in C l_{2}^{\geq}, \mathrm{r} 1$ is minimal among them, because it uses a weaker elementary condition than r2 (outcome 15 against 25);

- requiring in all elementary conditions the same cumulated outcome with less probability; for example, when considering two rules, $\mathrm{r} 4 \equiv$ if $\rho\left(a_{i}, 0.55\right) \geq 20$, then $a_{i} \in C l_{3}^{\geq}$, and $\mathrm{r} 5 \equiv$ if 
$\rho\left(a_{i}, 0.8\right) \geq 20$, then $a_{i} \in C l_{3}^{\geq}, \mathrm{r} 4$ is minimal among them, because it requires a cumulated outcome to be at least 20 , but with less probability, 0.55 against 0.8 .

Thus filtered, the remained conjunctions are used to construct the rules with a consequent: $a_{i} \in C l_{t}^{\geq}$. Let us denote by $\mathcal{R}_{\text {all }}^{\underline{\Pi}\left(\mathrm{Cl}_{t}^{\geq}\right)}\left(\mathcal{R}_{\text {all }}^{\underline{\Pi}\left(C l_{t}^{\leq}\right)}\right)$the set of all compatible minimal rules induced from $\underline{\Pi}\left(C l_{t}^{\geq}\right)$ $\left(\underline{\Pi}\left(C l_{t}^{\leq}\right)\right)$. Note that all compatible minimal rules are covering all consistent reference acts from the information table. In other words, they are supported by all classification examples being consistent with the stochastic dominance principle.

Table 2: All compatible certain minimal rules.

Symbol
$r_{\geq 2}^{1}$
$r_{\geq 2}^{2}$
$r_{\geq 2}^{3}$
$r_{\geq 2}^{4}$
$r_{\geq 2}^{5}$
$r_{\geq 2}^{6}$
$r_{\geq 3}^{1}$
$r_{\geq 3}^{2}$
$r_{\geq 3}^{3}$
$r_{\leq 1}^{1}$
$r_{\leq 1}^{2}$
$r_{\leq 1}^{3}$
$r_{\leq 1}^{4}$
$r_{\leq 2}^{1}$
$r_{\leq 2}^{2}$
$r_{\leq 2}^{3}$
$r_{\leq 2}^{4}$
$r_{\leq 2}^{5}$

Rule

if $\rho\left(a_{i}, 1.00\right) \geq 10$, then $a_{i} \in C l_{2}^{\geq}$

if $\rho\left(a_{i}, 0.55\right) \geq 20$, then $a_{i} \in C l_{2}^{\geq}$

if $\rho\left(a_{i}, 0.35\right) \geq 25$, then $a_{i} \in C l_{2}^{\geq}$

if $\rho\left(a_{i}, 0.20\right) \geq 30$, then $a_{i} \in C l_{2}^{\geq}$

if $\rho\left(a_{i}, 0.45\right) \geq 20$, and $\rho\left(a_{i}, 0.65\right) \geq 10$, then $a_{i} \in C l_{2}^{\geq}$

if $\rho\left(a_{i}, 0.45\right) \geq 20$, and $\rho\left(a_{i}, 1.00\right) \geq 5$, then $a_{i} \in C l_{2}^{\geq}$

if $\rho\left(a_{i}, 0.55\right) \geq 20$, then $a_{i} \in C l_{3}^{\geq}$

if $\rho\left(a_{i}, 0.20\right) \geq 30$, and $\rho\left(a_{i}, 1.00\right) \geq 10$, then $a_{i} \in C l_{3}^{\geq}$

if $\rho\left(a_{i}, 0.35\right) \geq 25$ and $\rho\left(a_{i}, 1.00\right) \geq 10$, then $a_{i} \in C l_{3}^{\geq}$

if $\rho\left(a_{i}, 1.00\right) \leq 0\left(\rho^{\prime}\left(a_{i}, 0.20\right) \leq 0\right)$, then $a_{i} \in C l_{1}^{\leq}$

if $\rho\left(a_{i}, 0.45\right) \leq 10\left(\rho^{\prime}\left(a_{i}, 0.65\right) \leq 10\right)$, then $a_{i} \in C l_{1}^{\leq}$

if $\rho\left(a_{i}, 0.35\right) \leq 20\left(\rho^{\prime}\left(a_{i}, 0.80\right) \leq 20\right)$ and $\rho\left(a_{i}, 1.00\right) \leq 5\left(\rho^{\prime}\left(a_{i}, 0.20\right) \leq 5\right)$, then $a_{i} \in C l_{1}^{\leq}$

if $\rho\left(a_{i}, 0.45\right) \leq 20\left(\rho^{\prime}\left(a_{i}, 0.65\right) \leq 20\right)$ and $\rho\left(a_{i}, 0.80\right) \leq 10\left(\rho^{\prime}\left(a_{i}, 0.35\right) \leq 10\right)$

and $\rho\left(a_{i}, 1.00\right) \leq 5\left(\rho^{\prime}\left(a_{i}, 0.20\right) \leq 5\right)$, then $a_{i} \in C l_{1}^{\leq}$

if $\rho\left(a_{i}, 1.00\right) \leq 0\left(\rho^{\prime}\left(a_{i}, 0.20\right) \leq 0\right)$, then $a_{i} \in C l_{2}^{\leq}$

if $\rho\left(a_{i}, 0.35\right) \leq 10\left(\rho^{\prime}\left(a_{i}, 0.80\right) \leq 10\right)$, then $a_{i} \in C l_{2}^{\leq}$

if $\rho\left(a_{i}, 0.35\right) \leq 20\left(\rho^{\prime}\left(a_{i}, 0.80\right) \leq 20\right)$, then $a_{i} \in C l_{2}^{\leq}$

if $\rho\left(a_{i}, 0.45\right) \leq 20\left(\rho^{\prime}\left(a_{i}, 0.65\right) \leq 20\right)$ and $\rho\left(a_{i}, 0.80\right) \leq 10\left(\rho^{\prime}\left(a_{i}, 0.35\right) \leq 10\right)$, then $a_{i} \in C l \leq$

if $\rho\left(a_{i}, 0.80\right) \leq 10\left(\rho^{\prime}\left(a_{i}, 0.35\right) \leq 10\right)$ and $\rho\left(a_{i}, 1.00\right) \leq 5\left(\rho^{\prime}\left(a_{i}, 0.20\right) \leq 5\right)$, then $a_{i} \in \mathrm{Cl}_{2}^{\leq}$

Support
$\left\{a_{1}, a_{13}\right\}$
$\left\{a_{1}, a_{3}\right\}$
$\left\{a_{1}, a_{3}, a_{18}\right\}$
$\left\{a_{1}, a_{3}, a_{18}\right\}$
$\left\{a_{1}, a_{3}, a_{12}, a_{18}\right\}$
$\left\{a_{3}, a_{18}\right\}$
$\left\{a_{1}, a_{3}\right\}$
$\left\{a_{1}\right\}$
$\left\{a_{1}\right\}$
$\left\{a_{2}\right\}$
$\left\{a_{7}\right\}$
$\left\{a_{2}, a_{7}\right\}$
$\left\{a_{2}, a_{7}\right\}$
$\left\{a_{2}\right\}$
$\left\{a_{2}, a_{7}, a_{13}, a_{18}\right\}$
$\left\{a_{2}, a_{7}, a_{13}\right\}$
$\left\{a_{2}, a_{7}, a_{13}\right\}$
$\left\{a_{2}, a_{7}, a_{18}\right\}$

Illustrative study: all compatible minimal rules. A set of all minimal decision rules induced from the lower approximations of quality class unions is provided in Table 2. There are 18 certain rules overall (9 rules for both the upward and downward class unions). When it comes to the number of elementary conditions, there are 10 rules with just a single condition, 7 rules with two conditions, and just a single rule with three elementary conditions. For each rule, we provide a subset of reference acts which are covered by it. This information will be used by an algorithm discussed in Section 5 to generate all minimal-cover sets of rules.

Satisfactory minimal rules. The algorithm presented in this section can be adapted to induce all minimal rules $\mathcal{R}_{\text {all }}^{\Pi\left(\mathrm{Cl}_{t}^{\geq}\right)}$that satisfy some pre-defined requirements. For example, we can account for the following requirements: 
- the minimal support $\sup _{r_{t}^{\geq}}^{\text {min }}$ of each rule by generating only these conjunctions of elementary conditions which cover at least $\sup _{r_{t}^{\geq}}^{\min }$ reference acts (instead of at least one) in $\underline{\Pi}\left(C l_{t}^{\geq}\right)$;

- the maximal $\Pi_{r_{t}^{\geq}}^{\max }$ number of probabilities involved in the rule (i.e., the maximal length of each rule) by stopping an iterative process once generated conjunctions of size $\prod_{r_{t}^{\geq}}^{\max }$.

\section{Generating All Compatible Minimal-Cover Sets of Rules}

A set of certain decision rules is minimal-cover if and only if it is complete, i.e., it is able to cover all reference acts, and non-redundant, i.e., exclusion of any rule from this set makes it non-complete. Finding a minimal set of rules covering the reference acts from $\underline{\Pi}\left(C l_{t}^{\geq}\right)\left(\underline{\Pi}\left(C l_{t}^{\leq}\right)\right)$is analogous to solving the minimum set covering problem. Thus, as noted in [22], a minimal-cover set of rules can be constructed using ILP (without loss of generality, we focus on $\underline{\Pi}\left(C l_{t}^{\geq}\right)$):

$$
\text { Minimize: } f_{w}=\sum_{\left.r_{k} \in \mathcal{R}_{\text {all }}^{\underline{\Pi}(C l} \stackrel{\geq}{t}\right)} v\left(r_{k}\right)
$$

s.t.

$$
\left.\begin{array}{l}
\sum_{r_{k} \text { covering } a_{i}} v\left(r_{k}\right) \geq 1, \text { for all } a_{i} \in A^{R}, \\
v\left(r_{k}\right) \in\{0,1\}, \text { for all } r_{k} \in \mathcal{R}_{\text {all }}^{\underline{\Pi}\left(C l_{t}^{\geq}\right)} .
\end{array}\right\} E_{\text {cover }}^{\text {minimal }}
$$

If $v\left(r_{k}\right)=1, r_{k}$ is used in the set of rules covering all reference acts from $\underline{\Pi}\left(C l_{t}^{\geq}\right)$in iteration $w=$ $1,2, \ldots$ The optimal solution of the above ILP (denoted with *; e.g., $f_{w}^{*}$ and $\left[v\left(r_{k}\right)^{*}, r_{k} \in \mathcal{R}_{\text {all }}^{\underline{\Pi}\left(\mathrm{Cl}_{t}^{\frac{⿱}{t}}\right)}\right]$ ) indicates one of the minimal-cover sets of rules:

$$
\mathcal{R} \underline{w}^{\Pi\left(C l_{t}^{\geq}\right)}=\left\{r_{k} \in \mathcal{R}_{\text {all }}^{\Pi\left(C l_{t}^{\geq}\right)} \text {, such that } v\left(r_{k}\right)^{*}=1\right\}
$$

Other sets can be identified by adding the constraints that forbid finding again the solutions which have been already identified in optimizations conducted in the previous iterations $(w, w-1, w-$ $2, \ldots, 1)$ :

$$
\sum_{\left.r_{k} \in \mathcal{R}_{w}^{\underline{\Pi}(C l} l_{t}\right)} v\left(r_{k}\right) \leq f_{w}^{*}-1
$$

Let us denote by $\mathcal{R}_{m}^{\Pi\left(C l^{\frac{⿱}{t}}\right)}\left(\mathcal{R} \frac{\Pi\left(C l_{t}^{\leq}\right)}{m r c}\right)$ all minimal-cover sets of rules for $\underline{\Pi}\left(C l_{t}^{\geq}\right)\left(\underline{\Pi}\left(C l_{t}^{\leq}\right)\right)$. All compatible minimal sets of rules $\mathcal{R}^{A^{R}}$ are formed by the following product:

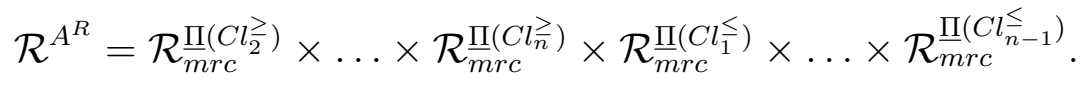

When computing each minimal-cover rule set in $\mathcal{R}^{A^{R}}$, we should eliminate decision rules from $\left.\mathcal{R}_{m}^{\frac{\Pi}{m}\left(\mathrm{Cl}_{\mathrm{t}}\right.}\right)$ or $\mathcal{R}_{m r c}^{\Pi\left(\mathrm{Cl}_{t}^{\leq}\right)}$with a consequent having at least the same strength (i.e., rules assigning objects to the

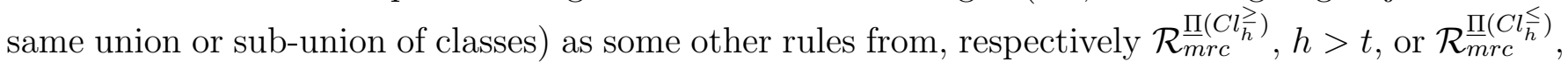
$h<t$. For example, when considering two rules, r6 $\equiv$ if $\rho\left(a_{i}, 0.65\right) \geq 20$, then $a_{i} \in C l_{3}^{\geq}$, and $\mathrm{r} 7 \equiv$ if 
$\rho\left(a_{i}, 0.65\right) \geq 20$, then $a_{i} \in C l_{2}^{\geq}, \mathrm{r} 6$ is minimal among them, because it suggests a stronger conclusion than $\mathrm{r} 7$, while using the same elementary conditions.

Illustrative study: all minimal-cover sets of rules. All minimal-cover sets of rules for the lower approximation of each class union are presented in Table 3. In particular, there are four minimalcover sets of rules for the reference acts from $\underline{\Pi}\left(C l_{2}^{\geq}\right)$, three sets of rules for $\underline{\Pi}\left(C l_{2}^{\leq}\right)$and $\underline{\Pi}\left(C l_{1}^{\leq}\right)$, and a unique way of covering all reference acts from $\underline{\Pi}\left(\mathrm{Cl}_{3}\right)$. Combination of these minimal rule covers leads to $36(=4 \times 1 \times 3 \times 3)$ compatible minimal-cover sets of minimal rules $\mathcal{R}^{A^{R}}$, which reproduce the consistent assignment examples provided by the DM. As a result, the number of rules in each of the sets is between four (e.g., $\left\{r_{\geq 2}^{5}, r_{\geq 3}^{1}, r_{\leq 1}^{3}, r_{\leq 2}^{2}\right\}$ ) and seven (e.g., $\left\{r_{\geq 2}^{1}, r_{\geq 2}^{3}, r_{\geq 3}^{1}, r_{\leq 1}^{1}, r_{\leq 1}^{2}, r_{\leq 2}^{3}, r_{\leq 2}^{5}\right\}$ ).

Table 3: All minimal-cover sets of rules for the lower approximations of class unions.

$$
\begin{aligned}
& \text { Minimal-cover sets of rules }
\end{aligned}
$$

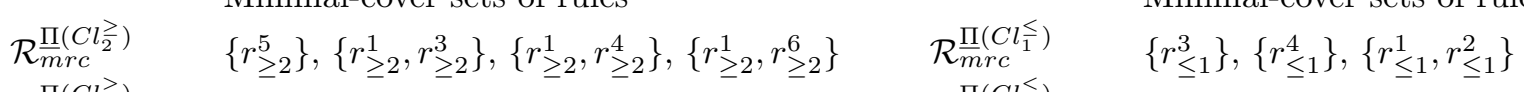

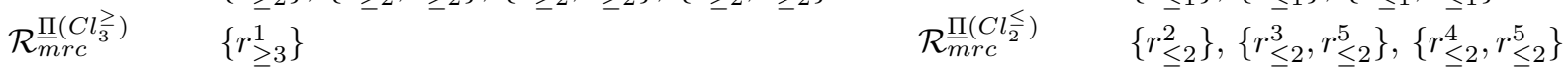

Satisfactory minimal-cover sets of rules. The algorithm presented in this section can be adapted to generate all minimal-cover sets of rules $\mathcal{R}_{m r c}^{\Pi\left(C l_{t}^{\geq}\right)}$that satisfy some additional requirements. For example, one can account for the following requirements by adding the constraints presented below to $E_{\text {cover }}^{\text {minimal }}$ :

- the minimal $N_{C l_{t}^{\geq}}^{m i n}$ and maximal $N_{C l_{t}^{\geq}}^{\max }$ number of rules:

$$
N_{C l_{t}^{\geq}}^{\min } \leq \sum_{r_{k} \in \mathcal{R}_{\text {all }}^{\underline{\Pi}(C l \stackrel{\geq}{t})}} v_{k} \leq N_{C l_{t}^{\geq}}^{\max }
$$

- the minimal $\Pi_{C l_{t}^{\geq}}^{m i n}$ and maximal $\Pi_{C l_{t}^{2}}^{m a x}$ number of probabilities (attributes) involved in the rules:

$$
\left.\begin{array}{l}
\Pi_{C l_{t}^{\geq}}^{\min } \leq \sum_{\pi_{i} \in \Pi} v\left(\pi_{i}\right) \leq \Pi_{C l_{t}^{\geq}}^{\max }, \\
v\left(\pi_{i}\right) \geq r_{k}, \text { for each } \pi_{i} \text { involved in } r_{k} \in \mathcal{R} \underline{a l l}^{\underline{\Pi}\left(C l_{t}^{\geq}\right)}, \\
v\left(\pi_{i}\right) \in\{0,1\}, \text { for all } \pi_{i} \in \Pi,
\end{array}\right\}
$$

where $v\left(\pi_{i}\right)$ is a 0-1 variable associated with using an elementary condition involving $\pi_{i} \in \Pi$; it is instantiated with 1 , once some rule $r_{k}$ referring to $\pi_{i}$ has been included in the minimal-cover set of rules; otherwise it gets a 0 value;

- the maximal $T_{C l_{t}^{\geq}}^{\max }$ number of rules that can be used to cover each reference act (if $T_{C l_{t}^{\geq}}^{\max }=1$, each reference act can be covered by a single rule only, which means that there is no redundancy 
with respect to covering of reference acts by the selected rules):

$$
\sum_{r_{k} \text { covering } a_{i}} v_{k} \leq T_{C l_{t}^{\geq}}^{\max }, \quad \text { for all } a_{i} \in A^{R}
$$

Note that some other requirements, e.g., concerning a number of rules used in each $\mathcal{R} \in \mathcal{R}^{A^{R}}$, an average rule length, or an average rule strength, cannot be satisfied at the phase of construction of minimal-cover sets of rules. Nevertheless, they can be accounted a posteriori, i.e., once all minimalcover sets of rules are generated.

\section{Robustness Analysis}

Each set of rules covering classification examples constitutes a single instance of the DM's preference model, and can be used to evaluate the non-reference acts $A \backslash A^{R}$. We apply the following sorting method [3, 22]. Let us denote by $l^{\mathcal{R}}\left(u^{\mathcal{R}}\right)$ the lowest (highest) class of the intersection of suggested unions by all $\mathrm{D}_{\geq^{-}}\left(\mathrm{D}_{\leq^{-}}\right)$decision rules in $\mathcal{R}$ covering $a$. If $l^{\mathcal{R}}$ and/or $u^{\mathcal{R}}$ are undefined or $l^{\mathcal{R}} \leq u^{\mathcal{R}}$, then a sorting procedure driven by a compatible set of rules $\mathcal{R}$ assigns an act $a \in A$ to an interval of classes $\left[C l_{L^{\mathcal{R}}(a)}, C l_{R^{\mathcal{R}}(a)}\right]$ such that

- $L^{\mathcal{R}}(a)=l^{\mathcal{R}}$, if $l^{\mathcal{R}}$ is defined, and $L^{\mathcal{R}}(a)=1$, otherwise;

- $R^{\mathcal{R}}(a)=u^{\mathcal{R}}$, if $u^{\mathcal{R}}$ is defined, and $R^{\mathcal{R}}(a)=n$, otherwise.

In case of inconsistency (i.e., if $u^{\mathcal{R}}<l^{\mathcal{R}}$ ), act $a$ is left without recommendation (i.e., the procedure indicates an empty set $\emptyset$ of classes).

\subsection{Class Assignments}

Since all compatible minimal-cover sets of rules are known, for each range of contiguous classes $\left[C l_{h_{L}}, C l_{h_{L}+1}, \ldots, C l_{h_{R}}\right]$, with $1 \leq h_{L} \leq h_{R} \leq n$, we can define class range acceptability index $C A I\left(a,\left[h_{L}, h_{R}\right]\right)$ as the share of compatible sets of rules $\mathcal{R} \in \mathcal{R}^{A^{R}}$ that assign act a precisely to the range of classes $\left[C l_{h_{L}}, C l_{h_{L}+1}, \ldots, C l_{h_{R}}\right]$. We can also compute the share of $\mathcal{R} \in \mathcal{R}^{A^{R}}$ for which $C l_{h}$ is within $\left[C l_{L^{\mathcal{R}}(a)}, \ldots, C l_{R^{\mathcal{R}}(a)}\right]$, i.e., the share of compatible sets of rules that either precisely or imprecisely assign $a$ to $C l_{h}$. Following [24], let us call such a share the cumulative class acceptability index $C u C A I(a, h)$.

Note that if $C u C A I(a, h)>0$, then $a$ is possibly assigned to $C l_{h}$ (let us denote it by $h \in C_{P}(a)$, and the extreme possible classes by $L_{P}(a)$ and $R_{P}(a)$ ), because there exists at least one compatible set of rules assigning $a$ to $C l_{h}$. If $C u C A I(a, h)=1, a$ is necessarily assigned to $C l_{h}$, because all compatible sets of rules assign $a$ to $C l_{h}$ (then $\left.h \in C_{N}(a)\right)$.

Illustrative study: class assignments. The class acceptability indices as well as the necessary and possible assignments are presented in Table 4 . For the six reference acts $\left(a_{1}, a_{2}, a_{3}, a_{7}, a_{13}, a_{18}\right)$ 
all compatible minimal-cover sets of rules reproduce the assignments provided by the DM. Thus, the necessary and possible assignments for these acts are non-empty and precise. Another seven nonreference acts $\left\{a_{8}, a_{9}, a_{10}, a_{14}, a_{15}, a_{17}, a_{20}\right\}$ are assigned precisely to a single class with all compatible minimal-cover sets of rules. There is also one act $\left(a_{6}\right)$ for which the possible assignment is imprecise, although the necessary one is non-empty. This means that with some minimal-cover sets of rules $a_{6}$ is assigned to $\left[C l_{1}, C l_{2}\right]$, while with the remaining ones to $C l_{2}$. As a result, $C_{P}\left(a_{6}\right)=\left[C l_{1}, C l_{2}\right]$ and $C_{N}\left(a_{6}\right)=C l_{2}$. For the remaining six non-reference acts, the necessary assignment is empty and the possible assignment is imprecise. This means that there is no agreement with respect to the recommendation between all compatible sets of rules. Three among these acts are possibly assigned to two consecutive classes, while the remaining three ones are assigned to any class between $C l_{1}$ and $\mathrm{Cl}_{3}$ depending on the choice of the compatible minimal-cover set of rules. Moreover, for these acts some models indicate $\emptyset$.

When it comes to the class acceptability indices, thirteen acts that are necessarily assigned to some class have the respective $C u C A I$ equal to $100 \%$. In general, for acts with imprecise possible assignments, we can analyze $C A I$ and $C u C A I$ to indicate a recommendation suggested by most of compatible minimal-cover sets of rules. For $a_{11}, 75 \%$ of compatible sets of rules suggest $C l_{1}$, while $\mathrm{Cl}_{2}$ is the most prevailing recommendation for $a_{19}$. On the other hand, for some other acts the shares of compatible minimal-cover sets of rules indicating different assignments are the same or close to each other. In particular, for $a_{5}$, more than $10 \%$ of compatible sets of rules suggest six different recommendations, ranging from precise assignments to $C l_{1}$ or $C l_{2}$, through imprecise indication of a set of two or three consecutive classes, to an empty set.

Table 4: Class acceptability indices $(C A I)$, cumulative class acceptability indices $(C u C A I)$, and possible $C_{P}$ and necessary $C_{N}$ assignments.

\begin{tabular}{|c|c|c|c|c|c|c|c|c|c|c|c|c|c|}
\hline \multirow[b]{2}{*}{ Act } & \multicolumn{7}{|c|}{$C A I$} & \multicolumn{4}{|c|}{$C u C A I$} & \multicolumn{2}{|c|}{ Assignments } \\
\hline & $1-1$ & $1-2$ & $1-3$ & $2-2$ & $2-3$ & $3-3$ & $\emptyset$ & 1 & 2 & 3 & $\emptyset$ & $C_{P}$ & $C_{N}$ \\
\hline$a_{1}$ & - & - & - & - & - & 100.0 & - & - & - & 100.0 & - & 3 & 3 \\
\hline$a_{2}$ & 100.0 & - & - & - & - & - & - & 100.0 & - & - & - & 1 & 1 \\
\hline$a_{3}$ & - & - & - & - & - & 100.0 & - & - & - & 100.0 & - & 3 & 3 \\
\hline$a_{4}$ & 33.3 & 11.1 & 5.6 & 11.1 & 5.6 & - & 33.3 & 50.0 & 27.8 & 11.1 & 33.3 & $1-3$ & - \\
\hline$a_{5}$ & 16.7 & 11.1 & 22.2 & 11.1 & 22.2 & - & 16.67 & 50.0 & 44.4 & 44.4 & 16.7 & $1-3$ & - \\
\hline$a_{6}$ & - & 25.0 & - & 75.0 & - & - & - & 25.0 & 100.0 & - & - & $1-2$ & 2 \\
\hline$a_{7}$ & 100.0 & - & - & - & - & - & - & 100.0 & - & - & - & 1 & 1 \\
\hline$a_{8}$ & 100.0 & - & - & - & - & - & - & 100.0 & - & - & - & 1 & 1 \\
\hline$a_{9}$ & 100.0 & - & - & - & - & - & - & 100.0 & - & - & - & 1 & 1 \\
\hline$a_{10}$ & - & - & - & - & - & 100.0 & - & - & - & 100.0 & - & 3 & 3 \\
\hline$a_{11}$ & 75.0 & - & - & - & - & - & 25.0 & 75.0 & - & - & 25.0 & 1 & - \\
\hline$a_{12}$ & 8.3 & 16.7 & - & 50.0 & - & - & 25.0 & 25.0 & 66.7 & - & 25.0 & $1-2$ & - \\
\hline$a_{13}$ & - & - & - & 100.0 & - & - & - & - & 100.0 & - & - & 2 & 2 \\
\hline$a_{14}$ & 100.0 & - & - & - & - & - & - & 100.0 & - & - & - & 1 & 1 \\
\hline$a_{15}$ & - & - & - & - & - & 100.0 & - & - & - & 100.0 & - & 3 & 3 \\
\hline$a_{16}$ & 16.7 & - & 33.3 & - & 33.3 & - & 16.7 & 50.0 & 33.3 & 66.7 & 16.7 & $1-3$ & - \\
\hline$a_{17}$ & - & - & - & - & - & 100.0 & - & - & - & 100.0 & - & 3 & 3 \\
\hline$a_{18}$ & - & - & - & 100.0 & - & - & - & - & 100.0 & - & - & 2 & 2 \\
\hline$a_{19}$ & 8.33 & 16.7 & - & 50.0 & - & - & 25.0 & 25.0 & 66.7 & - & 25.0 & $1-2$ & - \\
\hline$a_{20}$ & - & - & - & - & - & 100.0 & - & - & - & 100.0 & - & 3 & 3 \\
\hline
\end{tabular}




\subsection{Assignment-based Preference Relations}

For each pair of acts $a, b \in A$, we can define assignment-based pairwise outranking index, denoted by $\operatorname{APOI}(a, b)[24]$, as the share of compatible sets of rules $\mathcal{R} \in \mathcal{R}^{A^{R}}$ that assign act $a$ to a range of classes which is at least as good as the range of classes for act $b$, i.e., $L^{\mathcal{R}}(a) \geq L^{\mathcal{R}}(b)$ and $R^{\mathcal{R}}(a) \geq R^{\mathcal{R}}(b)$.

If some minimal-cover sets of rules indicate $\emptyset$, the acts with an empty assignment can be either excluded from the comparison (indeed, $\emptyset$ is incomparable with any class range), or when comparing a pair of acts, we may account only for these compatible minimal-cover sets of rules which provide a non-empty assignment for both acts. We will employ this procedure in our illustrative study.

Note that if $\operatorname{APOI}(a, b)>0$, then $a$ is possibly assigned to a class range at least as good as $b$ (let us denote it by $a \succsim_{P} b$ ). If $\operatorname{APOI}(a, b)=1, a$ is necessarily assigned to a class range at least as good as $b($ then $a \succsim \rightarrow \vec{N} b)$.

Illustrative study: assignment-based preference relations. A Hasse diagram of the necessary assignment-based preference relation is presented in Figure 2. The acts assigned to the same class range with all compatible minimal-cover sets of rules are indifferent in terms of $\succsim \vec{N}$, i.e.:

$$
\text { for all } a, b \in A \text { such that } C A I\left(a,\left[h_{L}, h_{R}\right]\right)=C A I\left(b,\left[h_{L}, h_{R}\right]\right)=100 \%, a \sim_{N} b \text {, }
$$

where $\sim_{N}$ is a symmetric part of $\succsim \vec{N}$. In particular, $a_{2}, a_{7}, a_{8}, a_{9}$, and $a_{14}$ are always assigned precisely to $C l_{1}\left(C A I\left(a_{i},[1,1]\right)=100 \%\right.$, for $\left.i=2,7,8,9,14\right)$, and, thus, they are related by the necessary indifference in terms of the assignment-based preference relation. Such a subset of acts forms a single node in Figure 2.

Further, pairs of acts which are not related by an arc in Figure 2 are incomparable in terms of $\vec{N}_{N}$, i.e., for some compatible minimal-cover sets of rules one of them is assigned to a class better than the other, whereas for some other minimal-cover sets the order of classes is inverse. For example, while $a_{13}$ is always assigned to $C l_{2}$ and $a_{16}$ is classified in $C l_{1}$ or $C l_{3}$ with some compatible minimal-cover sets, we have $\neg\left(a_{13} \succsim \vec{N} a_{16}\right)$ and $\neg\left(a_{16} \succsim \vec{N} a_{13}\right)$. As a general rule:

for all $a, b \in A$ such that $R_{P}(a)>R_{P}(b) \geq L_{P}(b)>L_{P}(a), \neg\left(a \succsim_{N} b\right)$ and $\neg\left(b \succsim_{N} a\right)$.

Finally, some other pairs of acts are related by the strict necessary assignment-based preference relation $\succ_{N}$, where $\succ_{N}$ is an asymmetric part of $\succsim_{N}$. This means that for all compatible minimalcover sets of rules one of acts is assigned to a class at least as good as the other, while the inverse statement is not true. In particular, $a_{10}$ is always assigned to a class strictly better than $a_{13}$, and, thus, $a_{10} \succ_{N} a_{13}$. In general:

$$
\text { for all } a, b \in A \text { such that } L_{P}(a)>R_{P}(b), a \succ_{N} b \text { and } \neg(b \succsim \vec{P} a) \text {. }
$$

Further, if the worst possible class for some act is not worse than the best possible class for another act 


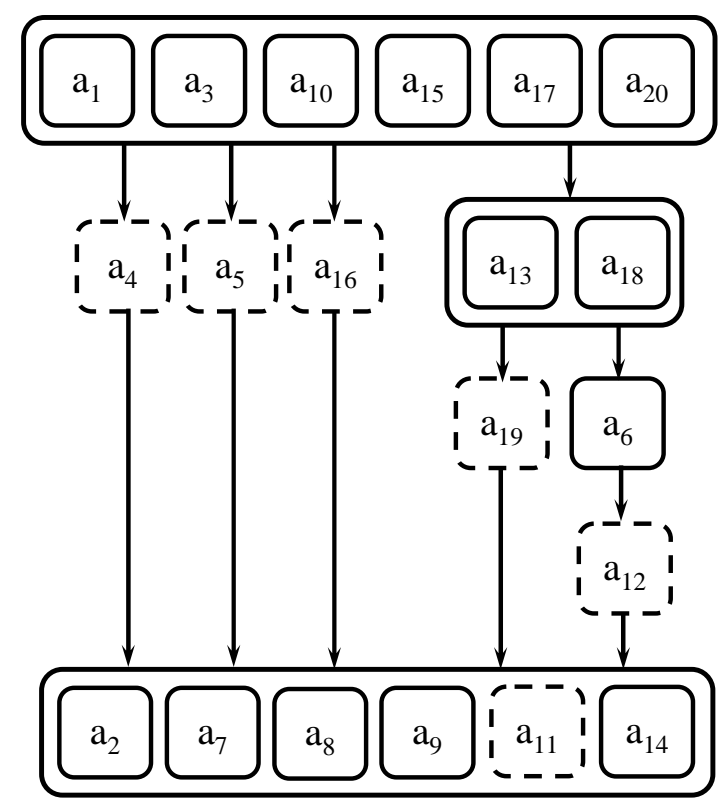

Figure 2: Hasse diagram (weak partial order) of the necessary assignment-based preference relation (dashed line indicates acts with empty assignment for at least one compatible set of rules).

with an imprecise possible assignment, this pair of acts is related by the strict necesary assignmentbased preference, i.e.:

$$
\text { for all } a, b \in A \text { such that } L_{P}(a) \geq R_{P}(b)>L_{P}(b), a \succ_{N} b \text {. }
$$

For example, for some compatible sets of rules both $a_{6}$ and $a_{18}$ are assigned to $C l_{2}$, whereas for some other compatible sets of rules $a_{6}$ is classified in $\left[\mathrm{Cl}_{1}, \mathrm{Cl}_{2}\right]$ while for $a_{18}$ still only $\mathrm{Cl}_{2}$ is possible. Thus, $L_{P}\left(a_{18}\right)=R_{P}\left(a_{6}\right)=2>L_{P}\left(a_{6}\right)=1$, and $a_{18} \succ_{N} a_{6}$. The same effect can be observed, e.g., for $\left(a_{1}, a_{4}\right),\left(a_{5}, a_{2}\right)$, or $\left(a_{12}, a_{7}\right)$. Nevertheless, $\succ \underset{N}{ }$ holds also for some other pair of acts. In particular, even though $C_{P}\left(a_{6}\right)=C_{P}\left(a_{12}\right), a_{6}$ is always assigned to a class at least as good as $a_{12}$, while the inverse statement is not true. Thus, $a_{6} \succ_{N} a_{12}$. This confirms that it is not possible to comprehensively infer $\succsim_{N}$ from $C A I s, C u C A I s$, and $C_{P}$.

\subsection{Class Cardinalities}

For each class $C l_{h}, h \in H$, we can define a class cardinality index $C C I(h, n)$ as the share of compatible minimal-cover sets of rules $\mathcal{R} \in \mathcal{R}^{A^{R}}$ that assign exactly $n$ acts to class $C l_{h}$ either precisely or imprecisely. In this perspective, it is particularly interesting to know the minimal $N_{h}^{\min }$ and maximal $N_{h}^{\max }$ cardinality of $C l_{h}$ defined as, respectively, the smallest and the greatest number of acts which are simultaneously assigned to $C l_{h}$ by some compatible set of rules $\mathcal{R} \in \mathcal{R}^{A^{R}}$, i.e.:

$$
N_{h}^{\min }=\min _{n}\{C C I(h, n)>0\} \text { and } N_{h}^{\max }=\max _{n}\{C C I(h, n)>0\}
$$

Illustrative study: class cardinalities. The class cardinality indices and extreme class cardinalities are presented in Table 5. For all classes, the cardinalities obtained with different compatible 
minimal-cover sets of rules are relatively stable, because $N_{h}^{\max }-N_{h}^{\min }=2$, for $h=1,2,3$. Note that these extreme cardinalities cannot be obtained directly from the analysis of the possible and necessary assignments. In particular, there are only five $\left(5<N_{1}^{\min }=7\right)$ acts necessarily assigned to $C l_{1}$ and twelve acts possibly assigned to $C l_{1}\left(12>N_{1}^{\max }=9\right)$. Thus, this analysis reveals how many acts are judged as good, medium, or bad simultaneously, i.e., for some compatible minimal-cover sets of rules.

Table 5: Class cardinalities indices $C C I$ (in \%) and extreme class cardinalities.

$\begin{array}{cccccccccccccc}n: & 0 & 1 & 2 & 3 & 4 & 5 & 6 & 7 & 8 & 9 & 10 & N_{h}^{\min } & N_{h}^{\max } \\ C l_{1} & 0.0 & 0.0 & 0.0 & 0.0 & 0.0 & 0.0 & 0.0 & 25.0 & 25.0 & 50.0 & 0.0 & 7 & 9 \\ C l_{2} & 0.0 & 0.0 & 0.0 & 0.0 & 0.0 & 33.3 & 33.3 & 33.3 & 0.0 & 0.0 & 0.0 & 5 & 7 \\ C l_{3} & 0.0 & 0.0 & 0.0 & 0.0 & 0.0 & 0.0 & 11.1 & 55.5 & 33.3 & 0.0 & 0.0 & 6 & 8 \\ \emptyset & 33.3 & 16.7 & 25.0 & 25.0 & 0.0 & 0.0 & 0.0 & 0.0 & 0.0 & 0.0 & 0.0 & 0 & 3\end{array}$

\section{Construction of a Univocal Recommendation}

The recent trend in MCDA consists in transforming outcomes of robustness analysis into a univocal recommendation. This stream of research is materialized either with the procedures for selecting a single representative instance of the preference model that would approximate the "true" model of the DM (see, e.g., [2, 8, 9, 21]), or with constructing a recommendation directly from the outcomes of robustness analysis, but without singling out a specific preference model instance [42]. In the context of DRSA, the procedures for selecting a representative minimal-cover set of rules have been discussed in [22]. In this section, we implement the other stream of research, additionally accounting for different types of indirect preference information.

As noted in [9], for each act an assignment to class with the highest acceptability can be considered as most robust with respect to the indirect preference information provided by the DM. Thus, when constructing a univocal sorting recommendation, it is reasonable to assign act $a$ to class $C l_{h}, h \in H$, such that for all $y \in H, C u C A I(a, h) \geq C u C A I(a, y)$. If there are no additional requirements, this can be achieved by selecting for each act individually a class with the maximal $C u C A I$. Nevertheless, we will formulate an optimization problem that will be subsequently extended so that to account for some additional requirements.

Let $v(a, h), a \in A, h \in H$, be a binary variable such that when being equal to one, then $a$ is assigned to class $C l_{h}$. Now, a univocal sorting recommendation can be constructed by solving the following MILP problem:

$$
\text { Maximize: } \delta+\varepsilon \sum_{a \in A} \sum_{h \in H} C u C A I(a, h) \cdot v(a, h)
$$

s.t. 


$$
\begin{aligned}
& \text { for each } a \in A \text { : } \\
& {\left[U N I_{1}\right] \sum_{h \in H} v(a, h)=1,} \\
& {\left[U N I_{2}\right] \delta \leq \sum_{h \in H} C u C A I(a, h) \cdot v(a, h), \quad E_{\text {assignment }}^{\text {univocal }}} \\
& \text { for each } h \in H, a \in A \text { : } \\
& {\left[U N I_{3}\right] v(a, h) \in\{0,1\} \text {, }}
\end{aligned}
$$

where $\varepsilon$ is an arbitrarily small positive value. We require each act to be assigned precisely to some class (see constraint $\left[U N I_{1}\right]$ ). As a primary criterion, we maximize $\delta$ which is equal to the lowest $C u C A I$ corresponding to an assignment selected for some act (see $\left[U N I_{2}\right]$ ). As a secondary criterion, we optimize the sum of $C u C A I$ corresponding to the assignments for all acts. Let us emphasize that instead of including the two criteria in the same MILP problem, we can optimize them in a lexicographic order. Alternatively, we can weight them so that to discover a solution with the best desired trade-off between the two objectives.

If there are no additional constraints, the other objective is consistent with the previous one. Thus, by maximizing $\sum_{a \in A} \sum_{h \in H} C u C A I(a, h) \cdot v(a, h)$, at the same time we maximize $\delta$. Nevertheless, we have already distinguished the two objectives, because when incorporating additional requirements to be satisfied by the univocal recommendation, we will first optimize $\delta$ representing the level of certainty for the collective assignment of all acts.

In the following subsections, we discuss preference modeling for two types of indirect preference information that may be considered apart from the classification examples. These are desired class cardinalities [23] and assignment-based pairwise comparisons [20]. The respective constraints are added to $E_{\text {assignment }}^{\text {univocal }}$ so that they are respected when constructing a univocal recommendation. As already announced in Section 1, the discussed optimization problems are more general, and can be combined with any approach delivering class acceptability indices (e.g., with some Monte Carlo simulation techniques $[20,41])$.

\subsection{Desired Class Cardinalities}

Desired class cardinalities consist of the minimal $N_{h, D M}^{\min }$ and/or maximal $N_{h, D M}^{\max }$ number of acts that can be assigned to class $C l_{h}, h \in H$, with $N_{h, D M}^{\min } \leq N_{h, D M}^{\max }$ [23]. Obviously, these requirements may refer to the proportions of the set of acts (e.g., 10\%,25\%, or 50\%) instead of explicit numbers. In any case, these limits may be modelled with the following constraints:

$$
\begin{aligned}
& \text { for each } C l_{h}, h \in H, \text { with specified desired cardinality: } \\
& \qquad[C L] \sum_{a \in A} v(a, h) \geq N_{h, D M}^{\min } \\
& {[C U] \sum_{a \in A} v(a, h) \leq N_{h, D M}^{\max }}
\end{aligned}
$$

Note that since $v(a, h)=1$ if $a$ is assigned to $C l_{h}, \sum_{a \in A} v(a, h)$ is equal to the number of acts in $A$ assigned to $C l_{h}$. 


\subsection{Assignment-based Pairwise Comparisons}

Assignment-based pairwise comparisons consist of two reference acts $\left(a^{*}, b^{*}\right) \in A^{R} \times A^{R}$ and imprecise comparison between their desired classes. Following [20], we account for the pairwise comparisons in the following forms:

- $a^{*}$ is better than $b^{*}$ by at least $k \geq 0$ classes, denoted by $a^{*} \succ_{\geq k, D M} b^{*}$;

- $a^{*}$ is better than $b^{*}$ by at most $l \geq 0$ classes, denoted by $a^{*} \underset{\leq l, D M}{\longrightarrow} b^{*}$;

- $a^{*}$ and $b^{*}$ represent the same class, denoted by $a^{*} \sim \overrightarrow{D M} b^{*}$.

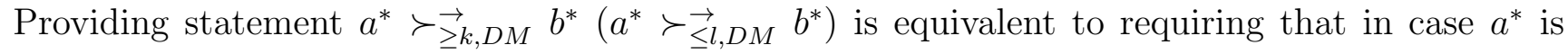
assigned to class at least (most) $C l_{h+k}\left(C l_{h+l}\right)$, then $b^{*}$ is assigned to class at most (at least) $C l_{h}$. As noted in [20], the character of these pairwise comparisons is imprecise, i.e., they do not refer directly to any specific classes. Thus, there exist $n-k$ or $n-l$ different combinations that need to be accounted as possible assignments for $a^{*}$ and $b^{*}$, and only one of them should be satisfied to ensure that the pairwise comparison is reproduced. These requirements can be modelled with the following set of constraints:

$$
\left.\begin{array}{l}
{[P C L] \text { for all } a^{*}, b^{*} \in A^{R}: a^{*} \succ_{\geq k, D M} b^{*}:} \\
\text { for } h=1, \ldots, n-k: \\
{\left[P C L_{1}\right] \sum_{i=h+k}^{n} v\left(a^{*}, i\right) \geq 1-v_{h}\left(a^{*}, b^{*}\right),} \\
{\left[P C L_{2}\right] \sum_{i=1}^{h} v\left(a^{*}, i\right) \geq 1-v_{h}\left(a^{*}, b^{*}\right),} \\
{\left[P C L_{3}\right] \sum_{h=1}^{n-k} v_{h}\left(a^{*}, b^{*}\right)=n-k-1,} \\
{\left[P C L_{4}\right] v_{h}\left(a^{*}, b^{*}\right) \in\{0,1\}, h=1, \ldots, n-k,}
\end{array}\right\} E^{P C L}
$$

and

$$
\left.\begin{array}{l}
{[P C U] \text { for all } a^{*}, b^{*} \in A^{R}: a^{*} \succ_{\leq l, D M} b^{*}:} \\
\text { for } h=1, \ldots, n-l: \\
\quad\left[P C U_{1}\right] \sum_{i=1}^{h+l} v\left(a^{*}, i\right) \geq 1-v_{h}\left(a^{*}, b^{*}\right), \\
{\left[P C U_{2}\right] \sum_{i=h}^{n} v\left(b^{*}, i\right) \geq 1-v_{h}\left(a^{*}, b^{*}\right),} \\
{\left[P C U_{3}\right] \sum_{h=1}^{n-l} v_{h}\left(a^{*}, b^{*}\right)=n-l-1,} \\
{\left[P C U_{4}\right] v_{h}\left(a^{*}, b^{*}\right) \in\{0,1\}, h=1, \ldots, n-l .}
\end{array}\right\} E^{P C U}
$$

For example, $\left[P C L_{1}\right]$ ensures that $a^{*}$ is assigned to a class at least $C l_{h+k}$, whereas $\left[P C L_{2}\right]$ guarantees that $b^{*}$ is assigned to a class at most $C l_{h}$. Finally, constraints $\left[P C L_{3}\right]$ and $\left[P C L_{4}\right]$ instantiate this scenario for some $h=1, \ldots, n-k$.

Finally, to ensure that $a^{*}$ and $b^{*}$ represent the same class $\left(a^{*} \sim_{D M} b^{*}\right), v\left(a^{*}, h\right)$ and $v\left(b^{*}, h\right)$ should be equal for all $h \in H$, i.e.:

$$
\left.\begin{array}{l}
{[P C I] \text { for all } a^{*}, b^{*} \in A^{R}: a^{*} \sim_{D M} b^{*}:} \\
\quad \text { for } h=1, \ldots, n: v\left(a^{*}, h\right)=v\left(b^{*}, h\right) .
\end{array}\right\} E^{P C I}
$$


Illustrative study: constructing a univocal recommendation. In Table 6, we present a univocal sorting recommendation for all acts in the three iterations of simulated interaction with the DM. In the first iteration, each act is assigned to a class with the highest $C u C A I$. In this case, the certainty of the collective assignment $\delta$ is equal to $50 \%$.

In the second iteration, we impose constraints on the class cardinalities. Precisely, we require that there are not less than six and not more than eight acts assigned to each class. This results in changing the assignment of $a_{5}$ from $C l_{1}$ to $C l_{2}$, and lowering $\delta$ to $44.4 \%$. The observed class cardinalities are equal to 7 for $C l_{1}$ and $C l_{3}$, and 6 for $C l_{2}$.

In the third iteration, we wish the univocal sorting recommendation to respect additional requirements in the form of three following assignment-based pairwise comparisons: $a_{4}$ and $a_{19}$ represent the same class, $a_{5}$ should be assigned to a class better than $a_{8}$, and $a_{4}$ should be assigned to a class at most as good as $a_{12}$. As a result, $a_{4}$ is moved to $C l_{2}$, thus increasing the cardinality of $C l_{2}$ to 7 , and decreasing $\delta$ to $27.8 \%$.

Table 6: Univocal recommendation (class indices and corresponding cumulative class acceptability indices) for the three iterations of interaction with the Decision Maker ( $\delta$ represents the certainty of the collective assignment).

\begin{tabular}{rcccccc} 
& \multicolumn{2}{c}{ Iteration I } & \multicolumn{2}{c}{ Iteration II } & \multicolumn{2}{c}{ Iteration III } \\
Act & Class & $C u C A I$ & Class & $C u C A I$ & Class & $C u C A I$ \\
$a_{1}$ & 3 & 100.0 & 3 & 100.0 & 3 & 100.0 \\
$a_{2}$ & 1 & 100.0 & 1 & 100.0 & 1 & 100.0 \\
$a_{3}$ & 3 & 100.0 & 3 & 100.0 & 3 & 100.0 \\
$a_{4}$ & 1 & $\mathbf{5 0 . 0}$ & 1 & 50.0 & 2 & $\mathbf{2 7 . 8}$ \\
$a_{5}$ & 1 & $\mathbf{5 0 . 0}$ & 2 & $\mathbf{4 4 . 4}$ & 2 & 44.4 \\
$a_{6}$ & 2 & 100.0 & 2 & 100.0 & 2 & 100.0 \\
$a_{7}$ & 1 & 100.0 & 1 & 100.0 & 1 & 100.0 \\
$a_{8}$ & 1 & 100.0 & 1 & 100.0 & 1 & 100.0 \\
$a_{9}$ & 1 & 100.0 & 1 & 100.0 & 1 & 100.0 \\
$a_{10}$ & 3 & 100.0 & 3 & 100.0 & 3 & 100.0 \\
$a_{11}$ & 1 & 75.0 & 1 & 75.0 & 1 & 75.0 \\
$a_{12}$ & 2 & 66.7 & 2 & 66.7 & 2 & 67.7 \\
$a_{13}$ & 2 & 100.0 & 2 & 100.0 & 2 & 100.0 \\
$a_{14}$ & 1 & 100.0 & 1 & 100.0 & 1 & 100.0 \\
$a_{15}$ & 3 & 100.0 & 3 & 100.0 & 3 & 100.0 \\
$a_{16}$ & 3 & 66.7 & 3 & 66.7 & 3 & 67.7 \\
$a_{17}$ & 3 & 100.0 & 3 & 100.0 & 3 & 100.0 \\
$a_{18}$ & 2 & 100.0 & 2 & 100.0 & 2 & 100.0 \\
$a_{19}$ & 2 & 66.7 & 2 & 67.7 & 2 & 67.7 \\
$a_{20}$ & 3 & 100.0 & 3 & 100.0 & 3 & 100.0 \\
$\delta$ & & $\mathbf{5 0 . 0}$ & & $\mathbf{4 4 . 4}$ & & $\mathbf{2 7 . 8}$ \\
& & & & & &
\end{tabular}

\section{Group Decision}

In this section, we propose some indicators and outcomes that may be computed to inform the DMs about the spaces of consensus and disagreement with respect to the classification of all acts. For the recent methodological advances in group decision making, see, e.g., [4, 5, 15, 19, 34]. 
For each DM, $D M_{k} \in \mathcal{D}=\left\{D M_{1}, \ldots, D M_{K}\right\}$, we consider her/his individual exemplary assignments of reference acts, and compute the cumulative class acceptability indices $C u C A I^{k}(a, h)$, as well as the possible $C_{P}^{k}(a)$ and necessary $C_{N}^{k}(a)$ assignments, as defined in Section 6.1.

In [7], a simple idea to compute the proportion of DMs that accept each possible assignment was given as:

$$
E^{\mathcal{D}}(a, h)=\frac{\sum_{k=1}^{K} E^{k}(a, h)}{K}
$$

where

$$
E^{k}(a, h)= \begin{cases}1 & \text { if } h \in C_{P}^{k}(a, h), \\ 0 & \text { otherwise. }\end{cases}
$$

The above measure accounts for the cumulative support given to the assignment of $a$ to $C l_{h}$. It can be extended to account for the degree of certainty that all minimal-cover sets of rules, being compatible with each DM's preference information, possibly assign $a$ to $C l_{h}$. Let us call such indicator a cumulative group class acceptability index $C u C A I^{\mathcal{D}}$, defined as follows:

$$
C u C A I^{\mathcal{D}}(a, h)=\frac{\sum_{k=1}^{K} C u C A I^{k}(a, h)}{K} .
$$

As suggested in [7], it is desirable to use a modified version for the computation of the above index that yields a unimodal distribution of its values over the range of possible assignments. Such a distribution excludes situations in which the support for some intermediate class $C l_{h}, 1<h<n$, is simultaneously lower than the support for some worse class $C l_{<h}$ and some better class $C l_{>h}$ than $C l_{h}$. It seems to be a natural proposal for consensus reaching. For example, a modified cumulative group class acceptability index can be defined in the following way:

$$
C u C A I^{\mathcal{D}}(a, h)= \begin{cases}C u C A I^{\mathcal{D}}(a, h) & \text { if } h=1 \text { or } h=n, \\ \max \left\{C u C A I^{\mathcal{D}}(a, h),\right. & \\ \min \left\{\max _{y<h}\left\{C u C A I^{\mathcal{D}}(a, h)\right\},\right. & \\ \left.\left.\max _{y>h}\left\{C u C A I^{\mathcal{D}}(a, h)\right\}\right\}\right\} & \text { otherwise. }\end{cases}
$$

Furthermore, when considering group decision, we can consider two levels of certainty for the results [12]. The first level is related to the necessary $(\mathrm{N})$ or possible $(\mathrm{P})$ consequences of indirect preference information provided by each DM, whereas the other level refers to the necessary $(\mathrm{N})$ or possible (P) agreement with respect to a set of DMs. This leads to defining four types of group assignments (below, we show what is their relation with the previously defined group indicators):

1. $C_{N, N}^{\mathcal{D}}(a)=\bigcap_{d_{k} \in \mathcal{D}} C_{N}^{k}(a)$, i.e., $h \in C_{N, N}^{\mathcal{D}}(a)$ iff $\forall d_{k} \in \mathcal{D}, h \in C_{N}^{k}(a)$ iff $C u C A I^{\mathcal{D}}(a, h)=1$;

2. $C_{N, P}^{\mathcal{D}}(a)=\bigcup_{d_{k} \in \mathcal{D}} C_{k}^{N}(a)$, i.e., $h \in C_{N, P}^{\mathcal{D}}(a)$ iff $\exists d_{k} \in \mathcal{D}, h \in C_{N}^{k}(a)$ iff $C u C A I^{k}(a, h)=1$;

3. $C_{P, N}^{\mathcal{D}}(a)=\bigcap_{d_{k} \in \mathcal{D}} C_{k}^{P}(a)$, i.e., $h \in C_{P, N}^{\mathcal{D}}(a)$ iff $\forall d_{k} \in \mathcal{D}, h \in C_{P}^{k}(a)$ iff $E^{\mathcal{D}}(a, h)=1$;

4. $C_{P, P}^{\mathcal{D} k}(a)=\bigcup_{d_{k} \in \mathcal{D}} C_{P}^{k}(a)$, i.e., $h \in C_{P, P}^{\mathcal{D}}(a)$ iff $\exists d_{k} \in \mathcal{D}, h \in C_{P}^{k}(a)$ iff $E^{\mathcal{D}}(a, h)>0$. 
For example, the non-empty necessary-necessary $(\mathrm{N}, \mathrm{N})$ assignment indicates the agreement with respect to the classification suggested by all minimal-cover sets of rules compatible with preference information provided by each individual DM.

Given the group indicators and assignments, the analyst may invite the DMs to a discussion. On the one hand, for acts with the non-empty possible-necessary (P-N) assignments with high support values $C u C A I^{\mathcal{D}}(a, h)$ reaching an agreement should be straightforward. On the other hand, the assignments with unacceptably low group indicator $C u C A I^{\mathcal{D}}(a, h)$ should be used to stimulate the DMs to revise some of their assignment examples or accept some number of "shifts" in their possible assignments. As defined in [7], one shift corresponds to changing an assignment of an act by one class above or below the range accepted by the DM.

Let us also note that the approach for constructing a univocal sorting recommendation, which was presented in Section 7, can also be used in the context of group decision. For this purpose, we just need to consider the group indicators $C u C A I^{\mathcal{D}}$ instead of $C u C A I$ obtained for an individual DM.

Illustrative study: group decision. Let us illustrate the computation of group indicators and assignments referring to the set of three DMs cooperating to make a collective decision. Each of them offers individual preference information composed of the exemplary assignments for some reference acts (see Table 7; note that preferences of $D M_{1}$ have been previously analyzed in Section 6). For each DM, we construct all compatible minimal-cover sets of rules and compute the cumulative class acceptability indices. These are presented in Table 8.

Table 7: Assignment examples provided by three Decision Makers.

$\begin{array}{rccc}\text { Class } & D M_{1} & D M_{2} & D M_{3} \\ C l_{1} & a_{2}, a_{7} & a_{4}, a_{8} & a_{11}, a_{14} \\ C l_{2} & a_{13}, a_{18} & a_{5}, a_{12} & a_{6}, a_{19} \\ C l_{3} & a_{1}, a_{3} & a_{3}, a_{15} & a_{10}, a_{17}\end{array}$

When it comes to the group assignments (see Table 8), the possible-possible assignments review all possible consequences of preference information of all DMs on sorting of the whole set of acts. For nine acts, these assignments are univocal, whereas only for four acts, each class between $C l_{1}$ and $\mathrm{Cl}_{3}$ is acceptable for at least one DM. Nevertheless, the ranges of possible-possible classes may be, in general, too wide to be decisive enough. Thus, an analyst and the DMs should rather analyze the possible-necessary assignments, which are formed by the intersection of the possible ranges of classes for all DMs. For our study, these assignments are precise for all acts but $a_{16}$ and $a_{6}$. For $a_{16}$, both $C l_{1}$ and $C l_{2}$ are acceptable by all DMs. On the contrary, for $a_{6}$, there is no agreement with respect to the assignment between $D M_{1}$ and $D M_{2}$. Thus, one of them should accept a single shift so that to reach a collective agreement for all acts.

The cumulative group acceptability indices $C u C A I^{\mathcal{D}}$ are relatively high (see Table 8; note that for out study $C u C A I^{\mathcal{D}}=C u C A I^{\prime \mathcal{D}}$ ). The worst among the maximal $C u C A I^{\mathcal{D}}$ analyzed for each act individually is equal to $64.8 \%$ for $C u C A I\left(a_{5}, 2\right)$. This value indicates the lowest certainty level 
for the whole group that has to be accepted by the three DMs if they wish to reach the consensus at the current stage of interaction with the method.

Table 8: Cumulative class acceptability indices $(C u C A I)$ for each individual DM and the whole group $\left(C u C A I^{\mathcal{D}}\right)$, and possible-possible $C_{P, P}$ and possible-necessary $C_{P, N}$ assignments.

\begin{tabular}{rccccccccccccccc}
\multicolumn{4}{c}{$C u C A I^{D M_{1}}$} & \multicolumn{4}{c}{$C u C A I^{D M_{2}}$} & \multicolumn{4}{c}{$C u C A I^{D M_{3}}$} & \multicolumn{3}{c}{$C u C A I^{\mathcal{D}}$} & \multicolumn{3}{c}{ Assignments } \\
Act & 1 & 2 & 3 & 1 & 2 & 3 & 1 & 2 & 3 & 1 & 2 & 3 & $C_{P, P}$ & $C_{P, N}$ \\
$a_{1}$ & - & - & 100.0 & - & 33.3 & 100.0 & - & 50.0 & 100.0 & - & 27.8 & $\mathbf{1 0 0 . 0}$ & $2-3$ & 3 \\
$a_{2}$ & 100.0 & - & - & 100.0 & - & - & 100.0 & - & - & $\mathbf{1 0 0 . 0}$ & - & - & 1 & 1 \\
$a_{3}$ & - & - & 100.0 & - & - & 100.0 & - & 50.0 & 50.0 & - & 16.67 & $\mathbf{8 3 . 3}$ & $2-3$ & 3 \\
$a_{4}$ & 50.0 & 27.8 & 11.1 & 100.0 & - & - & 100.0 & 50.0 & - & $\mathbf{8 3 . 3}$ & 25.9 & 3.7 & $1-3$ & 1 \\
$a_{5}$ & 50.0 & 44.4 & 44.4 & - & 100.0 & - & 100.0 & 50.0 & - & 50.0 & $\mathbf{6 4 . 8}$ & 14.8 & $1-3$ & 2 \\
$a_{6}$ & 25.0 & 100.0 & - & - & 100.0 & - & - & 100.0 & - & 8.3 & $\mathbf{1 0 0 . 0}$ & - & $1-2$ & 2 \\
$a_{7}$ & 100.0 & - & - & - & 100.0 & - & 100.0 & 50.0 & - & $\mathbf{6 6 . 7}$ & 50.0 & - & $1-2$ & - \\
$a_{8}$ & 100.0 & - & - & 100.0 & - & - & 100.0 & - & - & $\mathbf{1 0 0 . 0}$ & - & - & 1 & 1 \\
$a_{9}$ & 100.0 & - & - & 100.0 & - & - & 100.0 & - & - & $\mathbf{1 0 0 . 0}$ & - & - & 1 & 1 \\
$a_{10}$ & - & - & 100.0 & - & - & 100.0 & - & - & 100.0 & - & - & $\mathbf{1 0 0 . 0}$ & 3 & 3 \\
$a_{11}$ & 75.0 & - & - & 100.0 & - & - & 100.0 & - & - & $\mathbf{9 1 . 7}$ & - & & 1 & 1 \\
$a_{12}$ & 25.0 & 66.7 & - & - & 100.0 & - & - & 100.0 & - & 8.3 & $\mathbf{8 8 . 9}$ & - & $1-2$ & 2 \\
$a_{13}$ & - & 100.0 & - & - & 100.0 & - & 100.0 & 50.0 & - & 33.3 & $\mathbf{8 3 . 3}$ & - & $1-2$ & 2 \\
$a_{14}$ & 100.0 & - & - & 100.0 & - & - & 100.0 & - & - & $\mathbf{1 0 0 . 0}$ & - & - & 1 & 1 \\
$a_{15}$ & - & - & 100.0 & - & - & 100.0 & - & - & 100.0 & - & - & $\mathbf{1 0 0 . 0}$ & 3 & 3 \\
$a_{16}$ & 50.0 & 33.3 & 66.7 & 66.7 & 100.0 & - & 100.0 & 100.0 & - & 72.2 & $\mathbf{7 7 . 7}$ & 22.2 & $1-3$ & $1-2$ \\
$a_{17}$ & - & - & 100.0 & - & - & 100.0 & - & - & 100.0 & - & - & $\mathbf{1 0 0 . 0}$ & 3 & 3 \\
$a_{18}$ & - & 100.0 & - & - & 33.3 & 66.7 & - & 100.0 & 50.0 & - & $\mathbf{7 7 . 8}$ & 38.9 & $1-2$ & 2 \\
$a_{19}$ & 25.0 & 67.7 & - & - & 33.3 & 66.7 & - & 100.0 & - & 8.33 & $\mathbf{6 6 . 7}$ & 22.2 & $1-3$ & 2 \\
$a_{20}$ & - & - & 100.0 & - & - & 100.0 & - & - & 100.0 & - & - & $\mathbf{1 0 0 . 0}$ & 3 & 3
\end{tabular}

\section{Conclusions}

We have presented a framework for preference modeling and robustness analysis for decision under uncertainty. The whole approach is addressing situations where the acts are described by the outcomes which are gained with given probabilities. We consider an additive probability distribution defined over events, but we also show that the proposed method is valid for a probability with an ordinal qualitative scale. We assume the Decision Maker to provide exemplary classifications for reference acts. They are structured using Dominance-based Rough Set Approach. Then, we analyze the lower approximations of the unions of ordered quality classes, which contain non-ambiguous classification examples taking part in the construction of an aggregated preference model. This model has the form of all minimal-cover sets of certain rules reproducing the consistent assignments of reference acts. We also show how to construct satisfactory minimal-cover sets of rules which fulfill some pre-defined user's requirements.

We apply these multiple compatible sets of rules on a set of all acts, and draw robust conclusions about the classification. In particular, we analyze the certainty of assignments, assignment-based preference relations, and class cardinalities. In this regard, we refer to the necessary, possible, and extreme results, as well as to the shares of minimal-cover sets of rules confirming a specific recommendation. Then, we exploit the outcomes of robustness analysis to construct a univocal 
classification. At this stage, we account for the additional types of indirect preference information

in form of desired class cardinalities and assignment-based pairwise comparisons. Finally, we adapt the basic approach to group decision under uncertainty. We discuss a set of indicators and outcomes that may be used to judge the spaces of consensus between the Decision Makers with respect to the classification of different acts, and to organize a discussion for reaching a collective decision.

The whole approach can be extended in several ways, in particular, by:

- using other classification methods which suggest a precise assignment for each act (e.g., [3]) instead of possibly imprecise class interval as proposed in this paper;

- assigning weights to different sets of rules based on their characteristics;

- describing minimal-cover sets of rules in terms of multiple criteria, and using some interactive multiple-objective optimization methods for selecting a single set of rules that best corresponds to the user's requirements;

- accounting for consequences distributed over time [16];

- considering imprecise information on probabilities of the states of the world [31];

- proposing methods for robustness analysis for the decision under uncertainty formulated in terms of a multi-attribute ranking or choice problem [17].

\section{Acknowledgment}

The first two authors wish to acknowledge financial support from the Polish National Science Center. The authors thank three anonymous referees for their remarks which helped us to improve the paper.

\section{References}

[1] M. Allais. Le comportement de l'homme rationnel devant le risque; critique des postulats et axiomes de l'école américaine. Econometrica, 21:503-546, 1953.

[2] R. Bisdorff, P. Meyer, and T. Veneziano. Elicitation of criteria weights maximising the stability of pairwise outranking statements. Journal of Multi-Criteria Decision Analysis, 21(1):113-124, 2014.

[3] J. Błaszczyński, S. Greco, and R. Słowiński. Multi-criteria classification - a new scheme for application of dominance-based decision rules. European Journal of Operational Research, 181(3):1030-1044, 2007.

[4] S. Chakhar and I. Saad. Dominance-based rough set approach for groups in multicriteria classification problems. Decision Support Systems, 54(1):372-380, 2012. 
[5] F. Chiclana, J.M. Tapia Garca, M.J. del Moral, and E. Herrera-Viedma. A statistical comparative study of different similarity measures of consensus in group decision making. Information Sciences, 221:110-123, 2013.

[6] S. Corrente, S. Greco, M. Kadziński, and R. Słowiński. Robust ordinal regression in preference learning and ranking. Machine Learning, 93(2-3):381-422, 2013.

[7] S. Damart, L. C. Dias, and V. Mousseau. Supporting groups in sorting decisions: Methodology and use of a multi-criteria aggregation/disaggregation DSS. Decision Support Systems, 43(4):1464-1475, 2007.

[8] M. Doumpos and C. Zopounidis. Regularized estimation for preference disaggregation in multiple criteria decision making. Computational Optimization and Applications, 38(1):61-80, 2007.

[9] M. Doumpos, C. Zopounidis, and E. Galariotis. Inferring robust decision models in multicriteria classification problems: An experimental analysis. European Journal of Operational Research, 236(2):601-611, 2014.

[10] D. Ellsberg. Risk, Ambiguity, and the Savage Axioms. Quarterly Journal of Economics, 75:643$669,1961$.

[11] P.C. Fishburn. Nonlinear Preferences and Utility Theory. The John Hopkins University Press, 1988.

[12] S. Greco, M. Kadziński, V. Mousseau, and R. Słowiński. Robust ordinal regression for mul-

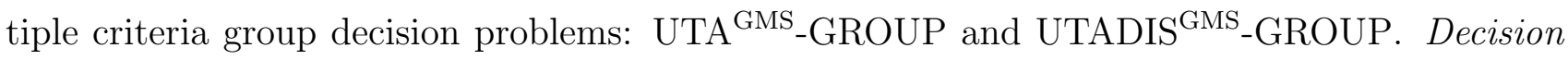
Support Systems, 52(3):549-561, 2012.

[13] S. Greco, M. Kadziński, and R. Słowiński. Selection of a representative value function in robust multiple criteria sorting. Computers $\& 6$ Operations Research, 38(11):1620-1637, 2011.

[14] S. Greco, B. Matarazzo, and R. Słowiński. Rough Sets Theory for Multicriteria Decision Analysis. European Journal of Operational Research, 129:1-47, 2001.

[15] S. Greco, B. Matarazzo, and R. Słowiński. Dominance-based rough set approach to decision involving a plurality of decision makers. In S. et al. Greco, editor, Rough Sets and Current Trends in Computing, volume 4259 of Lecture Notes in Artificial Intelligence, pages 306-317. Springer, 2006.

[16] S. Greco, B. Matarazzo, and R. Słowiński. Dominance-based Rough Set Approach to decision under uncertainty and time preference. Annals of Operations Research, 176:41-75, 2010.

[17] S. Greco, B. Matarazzo, and R. Słowiński. Dominance-based rough set approach to preference learning from pairwise comparisons in case of decision under uncertainty. In E. Hüllermeier, 
R. Kruse, and F. Hoffmann, editors, Computational Intelligence for Knowledge-Based Systems Design, volume 6178 of Lecture Notes in Computer Science, pages 584-594. Springer, 2010.

[18] S. Greco, V. Mousseau, and R. Słowiński. Multiple criteria sorting with a set of additive value functions. European Journal of Operational Research, 207(4):1455-1470, 2010.

[19] Y. Jiang, Z. Xu, and X. Yu. Group decision making based on incomplete intuitionistic multiplicative preference relations. Information Sciences, 295:33-52, 2015.

[20] M. Kadziński, K. Ciomek, and R. Słowiński. Modeling assignment-based pairwise comparisons within integrated framework for value-driven multiple criteria sorting. European Journal of Operational Research, 24(3):830-841, 2015.

[21] M. Kadziński, S. Greco, and R. Słowiński. Selection of a representative value function for robust ordinal regression in group decision making. Group Decision and Negotiation, 22(3):429-462, 2013.

[22] M. Kadziński, S. Greco, and R. Słowiński. Robust ordinal regression for dominance-based rough set approach to multiple criteria sorting. Information Sciences, 283:211-228, 2014.

[23] M. Kadziński and R. Słowiński. DIS-CARD: a new method of multiple criteria sorting to classes with desired cardinality. Journal of Global Optimization, 56(3):1143-1166, 2013.

[24] M. Kadziński and T. Tervonen. Stochastic ordinal regression for multiple criteria sorting problems. Decision Support Systems, 55(1):55-66, 2013.

[25] M. Kadziński, T. Tervonen, and J. Figueira. Robust multi-criteria sorting with the outranking preference model and characteristic profiles. Omega, (in press), 2014. http://dx.doi.org/10.1016/j.omega.2014.06.004.

[26] D. Kahnemann and A. Tversky. Prospect theory: an analysis of decision under risk. Econometrica, 47:263-291, 1979.

[27] H. Levy. Stochastic dominance: Investment decision making under uncertainty. Springer, New York, 2006.

[28] S. Li and T. Li. Incremental update of approximations in dominance-based rough sets approach under the variation of attribute values. Information Sciences, 294:348-361, 2015.

[29] M. Marinacci and L. Montrucchio. Introduction to the mathematics of ambiguity. In I. Gilboa, editor, Uncertainty in Economic Theory: a collection of essays in honor of David Schmeidlers 65th birthday, pages 46-107. Routledge, New York, 2004.

[30] Z. Pawlak. Rough Sets. Theoretical Aspects of Reasoning about Data. Kluwer Academic Publishers, 1991. 
[31] V.V. Podinovski. Decision making under uncertainty with unknown utility function and rankordered probabilities. European Journal of Operational Research, 239(2):537-541, 2014.

[32] T. Post and M. Kopa. General linear formulations of stochastic dominance criteria. European Journal of Operational Research, 230(2):321-332, 2013.

[33] L. Savage. The Foundations of Statistics. Wiley, New York, 1954.

[34] V.B.S. Silva and D.C. Morais. A group decision-making approach using a method for constructing a linguistic scale. Information Sciences, 288:423 - 436, 2014.

[35] R. Słowiński, S. Greco, and B. Matarazzo. Axiomatization of utility, outranking and decisionrule preference models for multiple-criteria classification problems under partial inconsistency with the dominance principle. Control and Cybernetics, 31(4):1005-1035, 2002.

[36] R. Słowiński, S. Greco, and B. Matarazzo. Rough set and rule-based multicriteria decision aiding. Pesquisa Operacional, 32(2):213-269, 2012.

[37] R. Słowiński, M. Kadziński, and S. Greco. Robust ordinal regression for dominance-based rough set approach under uncertainty. In M. Kryszkiewicz, C. Cornelis, D. Ciucci, J. Medina-Moreno, H. Motoda, and Z. Raś, editors, Rough Sets and Intelligent Systems Paradigms, volume 8537 of Lecture Notes in Computer Science, pages 77-87. Springer, 2014.

[38] C. Starmer. Developments in non-expected utility theory: the hunt for a descriptive theory of choice under risk. Journal of Economic Literature, 38:332-382, 2000.

[39] R. Susmaga. Reducts and constructs in classic and Dominance-based Rough Sets Approach. Information Sciences, 271:45-64, 2014.

[40] M. Szelag, S. Greco, and R. Słowiński. Variable consistency Dominance-based Rough Set Approach to preference learning in multicriteria ranking. Information Sciences, 277:525-552, 2014.

[41] T. Tervonen, J.R. Figueira, R. Lahdelma, J. Almeida Dias, and P. Salminen. A stochastic method for robustness analysis in sorting problems. European Journal of Operational Research, 192(1):236-242, 2009.

[42] R. Vetschera. Deriving rankings from incomplete preference information: A comparison of different approaches. In 20th Conference of the International Federation of Operational Research Societies, Barcelona, Spain, 2014.

[43] J. von Neumann and O. Morgenstern. The Theory of Games and Economic Behaviour. Princeton University Press, Princeton, 2nd edition, 1947.

[44] G.A. Whitmore and M.C. Findlay. Stochastic Dominance: An Approach to Decision- Making Under Risk. Lexington Books, Toronto, Canada, 1978. 
[45] H.-Y. Zhang, Y. Leung, and L. Zhou. Variable-precision-dominance-based rough set approach to interval-valued information systems. Information Sciences, 244:75-91, 2013.

\section{Appendix A. Notation Used Throughout the Paper}

In this section, we provide the meaning of all symbols used througout the paper.

Meaning Table A.9: Notation used in Section 2.

Probabilities

$z$ and $z^{\prime} \quad$ functions $z: A \times S \rightarrow \Pi$ and $z^{\prime}: A \times S \rightarrow \Pi$ assign a probability $\pi \in$ $\Pi$ to each pair composed of an act and a state

$z\left(a_{i}, s_{j}\right) \quad$ a probability of obtaining an outcome whose value is at least $g\left(a_{i}, s_{j}\right)$ by act $a_{i}$

$z^{\prime}\left(a_{i}, s_{j}\right) \quad$ a probability of obtaining an outcome whose value is at most $g\left(a_{i}, s_{j}\right)$ by act $a_{i}$

\section{Outcomes}

$\rho$ and $\rho^{\prime} \quad$ functions $\rho: A \times \Pi \rightarrow X$ and $\rho^{\prime}: A \times \Pi \rightarrow X$ assign an outcome to each pair composed of an act and a probability of an event

$\rho\left(a_{i}, \pi\right)=x \quad$ an outcome got by act $a_{i}$ is at least $x$ with a probability at least $\pi$

$\rho^{\prime}\left(a_{i}, \pi\right)=x \quad$ an outcome got by act $a_{i}$ is at most $x$ with a probability at least $\pi$

$C l_{t}^{\geq}$

Dominance-based Rough Set Approach

$C l_{t}^{\geq}$

an upward class union $C l_{t}^{\geq}=\bigcup_{s \geq t} C l_{s}$, for $t=2, \ldots, n$

a downward class union $C l_{t}^{\leq}=\bigcup_{s \leq t} C l_{s}$, for $t=1, \ldots, n-1$

$D_{\Theta} \quad$ stochastic dominance with respect to $\Theta \subseteq \Pi$

$D_{\Theta}^{+}\left(a_{i}\right)$

a set of acts dominating $a_{i}$ with respect to $\Theta \subseteq \Pi$

$D_{\Theta}^{-}\left(a_{i}\right)$

a set of acts dominated by $a_{i}$ with respect to $\Theta \subseteq \Pi$

$\underline{\underline{\Theta}}(X)$

$\Theta$-lower approximation of class union $X, X \in\left\{C l_{t}^{\geq}, C l_{t}^{\leq}\right\}$

$\overline{\bar{\Theta}}(X)$

$\Theta$-upper approximation of class union $X, X \in\left\{C l_{t}^{\geq}, C l_{t}^{\leq}\right\}$

Symbol Meaning

Table A.10: Notation used in Sections 4 and 5.

$\mathcal{R} \quad$ a single set of rules

$\mathcal{R} \frac{\Pi(X)}{\text { all }} \quad$ all minimal decision rules generated from the lower approximation of class union $X, X \in\left\{C l_{t}^{\geq}, C l_{t}^{\leq}\right\}$

$\mathcal{R}_{\text {all }}^{A^{R}} \quad$ all certain minimal decision rules

$\mathcal{R}_{m r c}^{\Pi(X)} \quad$ all minimal-cover sets of rules for $\underline{\Pi}(X), X \in\left\{C l_{t}^{\geq}, C l_{t}^{\leq}\right\}$

$\mathcal{R}^{A^{R}} \quad$ all minimal-cover sets of rules 
Symbol

$C A I\left(a,\left[h_{L}, h_{R}\right]\right)$

$C u C A I(a, h)$

$C_{P}(a)$

$C_{N}(a)$

$\operatorname{APOI}(a, b)$

$\succsim_{\vec{P}}$

$\succsim \vec{N}$

$C C I(h, n)$

$N_{h}^{\min }$

$N_{h}^{\min }$

Table A.11: Notation used in Section 6.

Meaning

a class range acceptability index for act $a$ and the range of classes $\left[C l_{h_{L}}, \ldots C l_{h_{R}}\right]$

a cumulative class acceptability index for act $a$ and class $C l_{h}$

a possible assignment for act $a$

a necessary assignment for act $a$

an assignment-based pairwise outranking index for a pair of acts $(a, b)$

a possible assignment-based preference relation

a necessary assignment-based preference relation

a class cardinality index for class $C l_{h}$ and cardinality $n$

a minimum cardinality of class $C l_{h}$

a maximum cardinality of class $C l_{h}$

Symbol Meaning

Table A.12: Notation used in Section 7.

$v(a, h) \quad$ a binary variable corresponding to an assignment of act $a$ to class $C l_{h}$

$N_{h, D M}^{\min } \quad$ a minimum number of acts that should be assigned to class $C l_{h}$ according to the DM

$N_{h, D M}^{\max } \quad$ a maximum number of acts that can be assigned to class $C l_{h}$ according to the DM

$\succ_{\geq k, D M}$ an assignment-based pairwise comparison indicating that one act should be assigned to a class better than another act by at least $k$ classes

$\succ_{\leq k, D M}^{\vec{a}}$ an assignment-based pairwise comparison indicating that one act should be assigned to a class better than another act by at most $k$ classes

$\sim \overrightarrow{D M} \quad$ an assignment-based pairwise comparison indicating that a pair of acts should be assigned to the same class

Symbol

$\mathcal{D}$

$E^{\mathcal{D}}(a, h)$

$C u C A I^{\mathcal{D}}(a, h)$

$\mathrm{CuCAI} I^{\prime \mathcal{D}}(a, h)$

$C_{N, N}^{\mathcal{D}}(a)$

$C_{N, P}^{\mathcal{D}}(a)$

$C_{P, N}^{\mathcal{D}}(a)$

$C_{P, P}^{\mathcal{D}}(a)$
Table A.13: Notation used in Section 8.

Meaning

a set of DMs, $\mathcal{D}=\left\{D M_{1}, \ldots, D M_{K}\right\}$

a proportion of DMs in $\mathcal{D}$ accepting the possible assignment of act $a$ to class $C l_{h}$

a cumulative group class acceptability index for act $a$ and class $C l_{h}$

a modified cumulative group class acceptability index for act $a$ and class $C l_{h}$

the necessary-necessary assignment for group $\mathcal{D}$ and act $a$

the necessary-possible assignment for group $\mathcal{D}$ and act $a$

the possible-necessary assignment for group $\mathcal{D}$ and act $a$

the possible-possible assignment for group $\mathcal{D}$ and act $a$ 